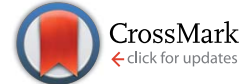

Cite this: J. Anal. At. Spectrom., 2017, 32,212

Received 7th September 2016 Accepted 2nd December 2016

DOI: $10.1039 / c 6 j a 00335 d$

www.rsc.org/jaas

\section{Improvements in the direct analysis of advanced materials using ICP-based measurement techniques}

\begin{abstract}
Andreas Limbeck, ${ }^{*}$ Maximilian Bonta and Winfried Nischkauer
Advanced materials are nowadays used in various industrial and scientific contexts, due to their particular and sometimes unique properties. In many cases, those properties are closely linked to the composition of the materials. An integral part in the characterization of advanced materials is therefore to determine their precise elemental composition, as well as to detect possible contaminants. With this information, the production as well as the properties of the final products can be optimized. To obtain such information in a routinely way, ICP-OES or ICP-MS are versatile tools, since those techniques allow sensitive multi-element analysis in a variety of matrices (e.g., high-purity materials, semiconductors, electronic components, metals, alloys, ceramics, and polymers). However, if using ICP-based techniques in their regular configuration, conversion of the solid material into a liquid solution is necessary. For this purpose, procedures such as acid digestion, fusion or dry ashing have been reported. However, although being well established, the application of these approaches is related with some drawbacks. Besides the problem of jeopardizing information on spatial distribution of analytes, some further shortcomings are risk of sample contamination and/or analyte losses, as well as increased time demand for sample preparation (especially in case of materials with high chemical resistance). Analyzing the solid sample directly is therefore an attractive alternative to conventional liquid analysis. Solid-sampling techniques which are frequently applied in combination with ICP-OES or ICP-MS detection are electro-thermal-vaporization (ETV) and laser-ablation (LA). Besides offering the mentioned advantages in sample preparation, solid-sampling techniques often allow for improvements in sensitivity, since unnecessary sample dilution could be avoided. Furthermore, LA (with restrictions also ETV) offers the possibility of spatially resolved analysis and depth profile analysis, providing information about the distribution of major, minor and trace constituents within
\end{abstract}

Institute of Chemical Technologies and Analytics, Division of Instrumental Analytical Chemistry, TU Wien, Getreidemarkt 9/164, A-1060 Vienna, Austria. E-mail: A.Limbeck@tuwien.ac.at; Fax: +43-1-58801-15199; Tel: +43-1-58801-15180

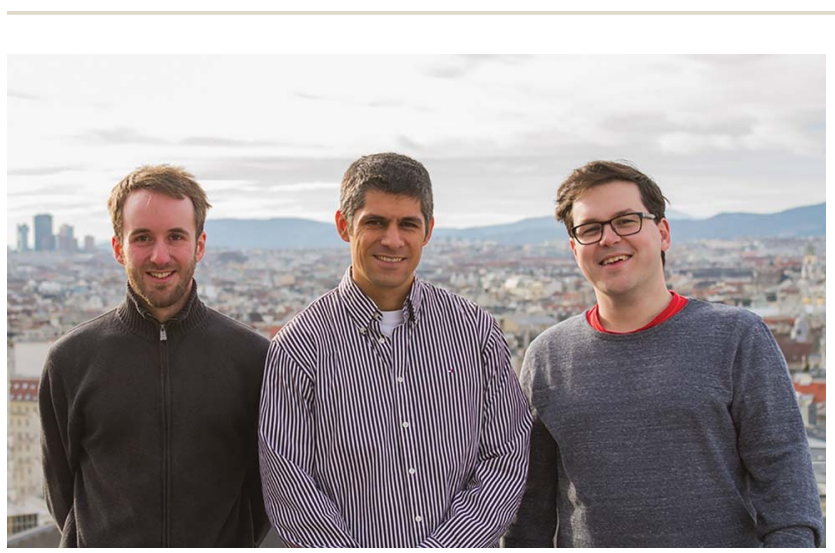

The authors presently work at the Institute of Chemical Technologies and Analytics at the TU Wien, Vienna, Austria. Andreas Limbeck (center) is assistant professor and currently head of the research division Instrumental Analytical Chemistry. His research interests are mainly focused on chemical analysis using laser-based solid sampling techniques (LA-ICP-MS/OES, LIBS) covering a wide application range from material science until the life sciences. Another research topic is environmental science and method development for improved sample analysis. Maximilian Bonta (left) finished his PhD in 2016, mainly working on LA-ICP-MS and LIBS for the (trace) elemental analysis of biological samples. Winfried Nischkauer (right) completed his $P h D$ in 2016. He developed concepts for the trace element analysis of challenging liquid samples using ICP-based analysis methods. During several side-projects of their PhD theses and in cooperation with industrial companies, both also gained valuable knowledge about the use of solid sampling techniques for investigations on advanced materials. 
the sample. The aim of this review is to discuss new analytical developments in ETV and LA in combination with ICP techniques for the quantitative determination of bulk, trace and ultra-trace elements in the routine analysis of advanced materials.

\section{Introduction}

Advanced materials have physical or chemical properties which set them apart from conventional materials. The term is often used in context with state-of-the-art developments such as nanomaterials, composites, photovoltaic devices, or electronic components. However, any material which emerges from a technological optimization process can be considered advanced, with respect to its original state. The scope of this review is to summarize latest developments in the quantitative characterization of advanced materials with regard to their bulk, trace and ultra-trace composition. Four groups of materials will be discussed: metals and alloys, ceramics and glasses, carboncontaining materials (polymers, graphite), and composite materials (layered systems or electronic components).

Optimizing the properties of a material is often related to determining its precise elemental composition and to monitor contaminants or dopants. In some cases, the average concentration in the material is a sufficient parameter (e.g., when performing quality-control of raw materials). In many cases, it is however necessary to perform laterally resolved analysis or to assess depth profiles of the final product. Nowadays, both tasks (bulk- and spatially resolved analysis) can be achieved using well-established techniques such as secondary ion mass spectrometry (SIMS), sputtered neutral mass spectrometry (SNMS), glow-discharge optical emission spectrometry (GD-OES), glow-discharge mass spectrometry (GD-MS), electron micro probe analysis (EMPA), X-ray photoelectron spectroscopy (XPS), auger electron spectroscopy (AES), transmission electron microscopy (TEM) or totalreflection X-ray fluorescence analysis (TXRF). ${ }^{1}$

General applicability of these techniques is sometimes limited by insufficient sensitivities (i.e., for X-ray based techniques). For the mass spectrometric techniques, where sensitivities are usually higher, low sample throughput and high instrument costs are major disadvantages. Moreover, the need of appropriate matrix matched standards or certified reference materials for quantitative determinations limit the applicability of the abovementioned techniques. ${ }^{2-4}$ Routine analysis of trace elements in advanced materials is therefore usually performed with atomic absorption spectrometry (AAS), inductively coupled plasma optical emission spectroscopy (ICP-OES) or inductively coupled plasma mass spectrometry (ICP-MS). Since AAS is basically a single-element method (although modern continuous source instruments can give access to more than one element simultaneously), the present review will focus exclusively on ICP-based techniques with their wide range of accessible elements and large dynamic range.

Since conventional application of ICP-OES and ICP-MS requires a liquid sample for analysis, solid materials have to be converted into liquids prior to measurement. For this purpose, several procedures have been reported. The decomposition of silicon carbide ceramics with concentrated $\mathrm{HF}, \mathrm{HNO}_{3}$ and $\mathrm{H}_{2} \mathrm{SO}_{4}$ is an example for the successful application of a microwave assisted digestion procedure to an advanced material. ${ }^{5}$ Alternatively, fusion or dry ashing of the sample in the presence of metal alkaline hydroxides, carbonates, or borates at elevated temperature is possible. ${ }^{6}$ More detailed information about typically applied pretreatment procedures involving dry dissolution, wet decomposition, and microwave-based methods can be found elsewhere. ${ }^{7,8}$

As outlined above, the dissolution of advanced materials often requires the use of highly corrosive/toxic reagents and/or harsh reaction conditions. The resulting solutions are often not compatible with analytical instrumentation, requiring further sample dilution. By doing so, trace and ultra-trace constituents might not be determined, as detection limits deteriorate with each dilution step. In addition, excessive sample-handling can increase blank levels. Also, applied reagents such as alkaline hydroxides may contribute to matrix-effects and can introduce contaminations causing additional interferences in sample analysis.

Solid sampling techniques in combination with ICP-OES or ICP-MS have therefore been employed for the direct analysis of advanced materials, circumventing the problems associated with sample dissolution. Such sampling techniques include slurry nebulization (SN), ${ }^{9,10}$ electrothermal vaporization (ETV), ${ }^{11,12}$ and laser ablation (LA). ${ }^{13,14}$ Briefly, $\mathrm{SN}^{15,16}$ and ETV ${ }^{17,18}$ are widely used for the direct analysis of powdered materials, and allow the use of aqueous calibration standards for signal quantification. In contrast to SN and ETV, LA provides also access to compact solid samples, even though quantitative analysis is much more challenging. But with this approach, information about the distribution of the analyte within the sample can be obtained (i.e., elemental mapping and depth profile analysis). ${ }^{19-22}$

This review will focus on recent developments in the analysis of advanced materials using ETV and LA in combination with ICP-based detection techniques. Slurry analysis is only discussed in combination with ETV or LA since Wang and Yang ${ }^{23}$ have recently reviewed conventional SN applications for advanced ceramics. The review is mainly based on the literature published in the last ten years (2005-2015), including novel and innovative concepts for sample analysis, such as dynamic etching procedures or LA of solids in liquid.

\section{Technical considerations regarding selection of the detection system in ETV or LA analysis}

When aiming at the direct analysis of advanced materials using ETV or LA coupled to an ICP-based detection method, a number 
of basic aspects have to be considered. One of the most fundamental ones is the appropriate choice of the detection system. With the selection between ICP-MS and ICP-OES, each method's characteristics have to be outlined. Typical parameters which should be taken into account are sensitivity, selectivity (spectral interferences), acquisition speed and susceptibility to matrix effects. While reproducibility of analysis can be considered similar when comparing the two detection techniques, one of the most prominent advantages of ICP-MS over ICP-OES is its higher detection power. Thus, with the use of ICP-MS as detection system, applications become possible which could not or only partially examined with ICP-OES. In addition to the determination of impurities at the ultra-trace level ICP-MS also allows the analysis of samples with restricted sample amounts such as single crystals or thin film coatings. Another benefit of higher detection power are improvements in depth profiling and laterally resolved analysis of advanced materials, since sensitivity of analysis determines the achievable resolution. However, although ICP-MS provides in most cases better detection limits, under certain circumstances ICPOES offers comparable or even higher sensitivity. Sensitivity of ICP-MS analysis is also affected by the presence of spectral interferences. Modern instrumentation offer measurements with increased mass resolution or the application of collision/ reaction cells to overcome most isobaric and/or polyatomic interferences. However, improvements achieved in selectivity are always accompanied by a decrease in the detection power. In contrast to ICP-MS, where flexibility of analysis is limited by the number of existing nuclides per element, detection with ICPOES provides a multitude of emission lines for each element. Thus, probably existing spectral interferences are less problematic, since usually analyte wavelengths can be selected in accordance with the needs in selectivity and/or sensitivity of the investigated research task. One example which demonstrates the sometimes prevailing limitations of ICP-MS in the direct analysis of advanced materials is the determination of calcium traces in strontium titanate $\left(\mathrm{SrTiO}_{3}\right)$ - a commonly used solid state electrolyte. In ICP-MS analysis, all calcium nuclides are interfered by doubly charged strontium ions or argon ions, hampering accurate determination. With the use of ICP-OES, analysis of $\mathrm{Ca}$ in $\mathrm{SrTiO}_{3}$ could be conducted without any restrictions. A general advantage of ICP-OES compared to ICPMS is the larger tolerance towards matrix load of the inductively coupled plasma. While this aspect is rather of importance in ETV analysis where the plasma load is considerably high, this might also be an issue in LA analysis, especially when the ablation rates are high. High matrix-load can influence the measurement in two ways: sudden introduction of a large sample amount into the plasma can lead to incomplete analyte ionization and/or excitation, which will influence accuracy of both ICPOES and ICP-MS analysis. During ICP-MS measurements, especially long analysis times can lead to a problematic amount of material deposited on the vacuum interface, altering the physical condition of the cones, causing instrumental drifts during measurement time. While material deposition is unproblematic in radial mode ICP-OES, a similar phenomenon can occur at the optical interface of axial mode ICP-OES measurements.
Thus, in most cases the apparent difference in data acquisition speed becomes the dominant selection criteria. Since the quality of ETV or LA measurements with ICP detection is strongly influenced by the acquisition speed, the complications related with this parameter will be described in more detail in the next chapter.

\section{Measurement and processing of short transient signals}

Although the working principles of ETV and LA are completely different, these two approaches have in common that the investigated solid sample is converted into a dry aerosol and subsequently transferred with a carrier gas into the ICP instrument. ${ }^{17,19,20,24}$ A brief description of these techniques is presented in the respective chapters, together with a discussion of the main challenges (aerosol formation, transport and atomization/excitation/ionization in the plasma). This section is focused only on the problems related to time-resolved multielement analysis of the aerosol introduced into the plasma.

When analyzing liquid samples, which is by far the most frequently applied measurement technique, an aerosol is produced from the liquid sample, and introduced at a constant rate into the plasma of the detecting ICP-OES or ICP-MS instrument. There, the introduced sample material is atomized, excited and ionized, resulting in analyte signals with more or less constant intensity. Such so-called steady-state signals are a precondition for measurements with long integration intervals, permitting the use of simultaneously as well as sequentially working ICP-spectrometers. Due to the steady-state nature of the signals, the analysis of several replicates from the same sample is possible. Moreover, signal stability can be improved and fluctuations of the background can be reduced, which significantly contributes to low detection limits. In case of ICPOES for signal acquisition, typical integration intervals of several seconds are used, resulting in total analysis times in the low minute range, depending on the demanded sensitivity and the number of measured replicates. ${ }^{25}$ In ICP-MS, the dwell times applied for the investigated mass-to-charge ratios $(\mathrm{m} / \mathrm{z})$ are generally in the order of below $10 \mathrm{~ms}$ to some $100 \mathrm{~ms}$. Considering settling times and the number of sweeps/replicates required per analyte, measurement times ranging from some seconds to tens of seconds are necessary for sample analysis. ${ }^{26}$ To summarize, conventional liquid sample introduction with a nebulizer results in constant plasma-load and therefore stable plasma conditions. As the observed signals show only minor variations, characteristic wavelengths or mass-to-charge ratios of the target elements can be measured sequentially. Furthermore, measurements with long integration/dwell times are possible, leading to improved reproducibility and sensitivity of analysis.

In contrast to conventional analysis of liquid samples with ICP-OES or ICP-MS, the use of ETV or LA for sample introduction requires a different measurement strategy. Since ETV offers no constant sample introduction rate, the amount of sample introduced into the plasma varies with time, resulting in 
transient signals with durations of typically 1-3 s for the investigated elements. ${ }^{\mathbf{1 8 , 2 4}}$ Transient signals with changing intensities are also generated when using LA for spatially resolved investigations (elemental mapping) and/or depth profile analysis. Independent of the applied ablation technique - single shot analysis or continuous ablation - measurement intervals of some hundred ms or below are necessary to monitor rapid signal changes, and to allow for sufficient lateral resolution or depth resolution, respectively. ${ }^{\mathbf{2 0 , 2 1}}$ To accomplish such short measurement intervals, simultaneous detection of the analytes of interest, fast data acquisition, readout and storage are desired. Furthermore, sudden changes in the plasma load have to be considered, which may result in fluctuating temperature and electron number density in the ICP. Thus, the robust and stable plasma conditions prevailing during conventional liquid analysis do not exist when the amount and composition of the sample material introduced into the plasma changes rapidly. Although the effect of changing plasma conditions can be reduced with the use of an appropriate internal standard, analyte detection and data treatment require methodical but also instrumental improvements to overcome existing limitations.

\section{Measurement of short transient signals using ICP-OES}

In the past, different types of ICP-OES spectrometers have been established for the analysis of liquid samples. ${ }^{27}$ Based on the way of light dispersion and detection, so-called sequential systems and simultaneous systems can be differentiated. Sequential spectrometers consist of a monochromator/detector unit arranged in the Czerny-Turner geometry - as prevailing in most atomic-absorption-spectrometers - and measure the characteristic wavelengths of the investigated elements successively. Thus, multi-element analysis of short transient signals is not possible with this type of instrument. Simultaneous spectrometers allow measurement of several lines or even the whole emission spectrum at the same time. This goal can be accomplished by either using polychromators in the PaschenRunge optical mounting or echelle-based dispersive systems. Although in the past commercial Paschen-Runge systems were equipped with photomultiplier tubes (PMT) positioned on the Rowland-circle, nowadays for both systems only charge transfer device detectors are used (i.e., charge coupled device (CCD) and charge injected device (CID)). The main advantage of CCD or CID detectors over PMT is that they provide the possibility to analyze signal intensities of analytes and background simultaneously. Furthermore, more flexibility in analytical line selection, compensation of matrix effects (internal standardization) and correction of spectral interferences is offered. However, besides simultaneous detection of emission spectra some additional prerequisites must be fulfilled for measurement of transient signals. For example, the speed of data acquisition is important, since the usually applied integration intervals of several seconds exceed the duration of short transient signals. With modern instrumentation, integration intervals of $0.1 \mathrm{~s}$ are possible, thus the course of a typical ETV signal can be described with 10 to 20 data points. However, such a high sampling rate results in poor signal stability and poor signal to noise ratio. Thus, in most works, integration intervals in the order of 0.3 to $0.5 \mathrm{~s}$ were used as a compromise between high time resolution and sufficient precision of analysis. As a consequence of the low data handling speed it is not possible to measure the full wavelength spectrum when using such short integration intervals. Rather, exact positions of the emission lines of interest and settings for background corrections have to be defined prior to measurement, which is a difficult task since background can change substantially during ETV measurement. Obtaining valid analytical results demands therefore measurement of two or more emission lines for each analyte of interest. At this point it has to be mentioned that increased analyte concentrations and/or too long integration times may cause saturation of the CCD detectors. Selection of multiple appropriate wavelengths is therefore important. Particularly for the analysis of unknown samples, the selection of lines with different sensitivities (covering a wide concentration range) is recommended to ensure useful results. A general protocol for multi-element analysis of short transient signals using an ICPOES instrument equipped with a CCD detector system (Spectro Arcos) has been described by Chaves et al. ${ }^{28}$

Evaluation and further processing of derived data is another challenging task. Currently available software is designated for measurement of steady state signals; only some instrument manufacturers provide software capable of recording transient signals. In contrast to the conventional measurement mode, this acquisition mode for transient signals provides only information about predefined parts of the emitted spectrum. Possibilities for background and/or interference correction are therefore limited. Furthermore, software features intended for data processing are rather simplistic, thus in most cases derived data has to be exported for further treatment with appropriate software (e.g., chromatography packages). In contrast to ICPOES software, recent ICP-MS software often contains the required features to evaluate transient signals, probably because coupling of separation techniques such as gas chromatography, liquid chromatography or electrophoresis with ICP-MS has become very popular in recent years. Ongoing improvements in ICP-OES should therefore focus on data processing to take full advantage of the information provided, as has been recently demonstrated by Spectro Ametek with the release of a special software package for their Arcos-System, which allows measurement of transient signals with fully automated data treatment. Furthermore, advances in data acquisition are required - fast monitoring of the full spectral range with sufficient resolution should be enabled. Data transfer rates must be increased to allow faster processing.

\section{Measurement of short transient signals using ICP-MS}

As for ICP-OES measurements, the simultaneous measurement of all target analytes is a prerequisite for accurate multi-element analysis of typical ETV or LA signals with ICP-MS. ${ }^{29}$ From the four types of mass spectrometers currently commercially available in combination with an ICP as ion source, quadrupole (QMS), time of flight (TOFMS), scanning sector field (SFMS) and 
Mattauch-Herzog sector field mass spectrometer, only the last one provides the possibility to measure the entire mass spectrum simultaneously. However, for this new kind of ICP-MS published literature is sparse, especially information about the measurement of transient signals is missing. Therefore, further discussion will focus on the three more common types (QMS, SFMS and TOFMS).

With QMS and SFMS systems, only a single nuclide can be monitored at a given time, thus for multi-element analysis selected $\mathrm{m} / \mathrm{z}$ ratios have to be measured one after another. The time spent for analysis of a certain $\mathrm{m} / \mathrm{z}$ is called "dwell time", whereas the "settling time" describes the amount of time required for the electronics to stabilize before the next $\mathrm{m} / \mathrm{z}$ measurement could be performed. Due to the short duration of the signals produced by ETV and LA, sequential scanning of the entire range of $\mathrm{m} / \mathrm{z}$ values and thus measurement of full mass spectra is not possible with QMS and SFMS instrumentation. As a consequence, the so-called "peak jumping mode" (sometimes also referred to as peak hopping mode) is normally applied, using short dwell times (typical values are 10-20 ms) for a limited set of $\mathrm{m} / \mathrm{z}$ ratios. Since quality of analysis (precision, accuracy) depends on the time required to complete one cycle of measurements for all selected $\mathrm{m} / \mathrm{z}$ ratios (in literature denoted as cycle time or scan speed), in most investigations the number of monitored isotopes has been restricted. In ETV applications typically a maximum number of 10-12 nuclides have been used per analysis, ${ }^{24}$ in case of LA typical values ranging from 8-15 were reported, ${ }^{\mathbf{1 9 2 1 , 2 2}}$ for isotopic analysis values are usually lower. In literature two strategies are described to overcome this limitation in the multi-element analysis of short transient signals - the use of faster data acquisition rates or broadening of the signal to provide more time for analysis. For alteration of ETV signals several methodical approaches have been published, for example the use of transient extension (TEx) chambers ${ }^{30}$ or single bead string reactors. ${ }^{31}$ In contrast to these methodical improvements, higher data acquisition rates require certain instrumental developments. Recent advances in quadrupole technology and data processing techniques provided a distinct reduction of instrument dwell times from ms down to tens of $\mu$ s, enabling the use of ICP-QMS for characterization, sizing, and quantification of metal-based nanoparticles. $^{32}$ The transient signals generated by the analysis of such single nanoparticles are less than about $0.5 \mathrm{~ms}$ in length, thus their detection requires the use of instruments capable of very fast data acquisition at greater frequencies of $10^{4}-10^{5} \mathrm{~Hz}{ }^{33}$ However, a precondition for single-particle investigations with ICP-QMS is the selection of only one isotope for analysis. Thus, measurement of composites (e.g. core-shell particles) or unknown samples is not feasible with this approach. Multielement investigations still require sequential monitoring of the selected isotopes, necessitating finite settling times between the different $m / z$ ratios, resulting in decreased data acquisition rates and data acquisition lags between $\mathrm{m} / \mathrm{z}$ changes. Nevertheless, achieved instrumental improvements (shorter dwell times, faster data acquisition and processing) are considered to be advantageous for analysis of transient signals generated by ETV or LA.
A more promising approach for multi-element analysis of fast transient signals is the use of ICP-TOFMS instrumentation. In contrast to QMS and SFMS which need hundreds of ms for full mass spectra acquisition, TOFMS generates complete mass spectra in distinctly shorter time. Basically in TOFMS nuclides with different $\mathrm{m} / \mathrm{z}$ values are also sequentially measured. However, as un-separated ion packages are collected and subsequently injected into the mass analyzer with very high scan speed, typically several thousand scans are performed per second. TOFMS has to be considered as a quasi-simultaneous measurement technique. The first commercial ICP-TOFMS instruments enabled complete mass spectra acquisition with time resolutions in the order of $10 \mathrm{~ms}$ only. ${ }^{34,35}$ Averaging of spectra and restrictions in data readout prevented shorter intervals. Nevertheless transient signals with durations of few hundred milliseconds were measured without limitations in multi-element detection. In the meanwhile, distinct improvements in data acquisition and handling were achieved, resulting in full mass spectra measurements within $30 \mu$ s only. ${ }^{36,37}$ So far these instruments have been successfully applied for the analysis of metal-labeled biomarkers in single cells, ${ }^{38}$ as well as for the simultaneous investigation of nanoparticles with different composition. ${ }^{39}$ Although scanning properties and data handling are superior when compared to conventional QMS and SFMS instrumentation, higher background, lower sensitivity, and a narrower dynamic range might prohibit some applications. Nevertheless, for tasks where sensitivity does not matter, distinct improvements in the recording of transient signals can be achieved with TOF instruments. For example, preliminary definition of the nuclides to be measured and thus knowledge about sample composition is not necessary, since ICP-TOFMS provides access to full mass spectra. This allows simpler identification of unknown sample constituents.

\section{Methods using electro thermal vaporization coupled to ICP devices}

This chapter will describe instrumental and operational details of ETV, as well as give an overview of recent developments in unconventional ETV approaches such as thermal resolution and thermal speciation. Quantitation in the context of ETV will be addressed, with focus on recent developments such as nonquantitative fingerprinting for forensic purposes.

\section{General principle and possibilities for sample introduction}

In ETV, a defined amount of sample is brought into a graphite boat, which is then introduced into the graphite furnace, where it is subjected to a temperature program for pyrolysis of the sample matrix, followed by the release of the analyte into the gas phase. Those vapors are then directed to the ICP torch using argon as transport gas. To the best of the author's knowledge, today there is only one commercial provider of ETV systems (ETV 4000 from Spectral Systems), but discontinued systems of the HGA series which was formerly produced by Perkin Elmer are still functional in many labs. Also custom-built models exist, as for example tungsten or rhenium wire vaporization systems, 
or systems using halogen lamps, or modified graphite furnaces from atomic absorption systems (see Tables 1 and 2). Although commercial systems might be more practical in terms of handling, robustness, and technical support, custom-built devices allow tackling of non-routine applications. For example, replacing the graphite furnace by a tungsten-coil set-up avoids carbon-based spectral interferences in ICP-MS (e.g., ${ }^{40} \mathrm{Ar}^{12} \mathrm{C}^{+}$on $\left.{ }^{52} \mathrm{Cr}^{+}\right) .{ }^{40-42}$ Besides being carbon-free, metal furnaces can also allow for higher heating rates than graphite systems, which gives rise to sharper transient signals and better $\mathrm{S} / \mathrm{N}$ ratios. Kataoka et $a l .{ }^{43}$ have intensified this feature by heating a tungsten platform to $2300{ }^{\circ} \mathrm{C}$ and holding a steel sample with a magnetized steel needle just above the hot platform. As soon as the steel sample is heated above its Curie temperature, the magnet no longer holds the sample, and it drops onto the hot platform, providing instantaneous vaporization of the analytes and very sharp transient signals. Although the authors discuss the improved sensitivity arising from such sharp signals, the rapid changes in ICP-load might be a drawback in terms of nonspectral interferences. Moreover, as discussed in the previous chapter, data acquisition of extremely short transient signals is a challenge, if more than one element is analyzed with sequential analyzers (QMS or SFMS). Just to avoid such swift signal development, and to obtain best results for tungsten isotope ratios, Okabayashi et $a{ }^{44}$ have deliberately used a slow heating ramp when using a rhenium wire ETV. As discussed in more detail in the section about transient signal processing, careful tradeoff between sensitivity and signal stability (repeatability) must be considered for every combination of analyte, matrix and detector.

Introducing the solid sample into the ETV oven can be achieved in two ways: either by weighing the dry material into the boats, or by first producing a suspension of the solid material in a suitable solvent, then pipetting a sub-sample of the suspension into the boat, and evaporating the liquid fraction. The first approach is referred to as solid sampling ETV, whereas the second one is referred to as slurry sampling ETV. We will discuss the advantages and disadvantages of both approaches separately, and in the context of the analysis of functional materials.

Solid sampling ETV is a very straight-forward approach, since there are no strict limitations in terms of powder characteristics. Even the analysis of compact pieces of the sample can be carried out, as has been demonstrated by the analysis of paint chips ${ }^{45}$ from automotive cars for forensic applications. This allows to work with samples where only a very small amount is available, and grinding the material into a homogeneous powder is no option. Also metals are difficult to pulverize, which might be the reason why practically all ETV-ICP methods for the analysis of metal matrices use the solid sampling approach (see Tables 1 and 2). However, introducing dry powder into small graphite boats is a tedious process and requires experience. One way to better distribute a fine, dry powder across the bottom of the graphite boat, was shown by Hassler et al. $^{\mathbf{4 6}}$ After weighing the dry material in the boat, the authors added a drop of ethanol onto the powder. This helped to distribute the powder inside the graphite boat more homogeneously, and resulted in more uniform vaporization of the sample. To reduce manual weighing (and the errors related to it), automated powder dispensing and weighing systems can help during sample introduction (e.g., as offered by Spectral Systems for the ETV 4000). In solid sampling ETV, the sample mass introduced into one boat is typically in the range of a few milligrams (see Table 1) and is often dictated by the readability of the analytical balances used. Using this solid sampling approach, it is also possible to use distinctly larger sample masses (e.g., significantly above a few milligrams). ${ }^{\mathbf{1 1 , 4 7}}$ Although larger sample intakes allow to improve sensitivity, the presence of elevated levels of bulk material might lead to increased matrix effects.

Slurry sampling avoids problems potentially related to introducing small amounts of dry powder into graphite boats (readability of balance, static electric charging of dry powders). Furthermore, sample homogeneity is less important since usually higher sample intakes are used for slurry preparation, thereby improving representativeness of analysis. To obtain a slurry, the powdered solid is suspended in a liquid and homogenized. Concentrations of slurries are typically in the order of $1 \%(\mathrm{~m} / \mathrm{v})$ powder in the liquid, but for special applications even higher sample contents were reported. ${ }^{15,16}$ To obtain slurries which are stable over longer time, and to avoid flocculation or particle sedimentation, the addition of stabilizing agents such as surfactants, wetting agents or dispersants is often performed (see Table 2). Frequently applied stabilizing agents include diluted inorganic acids, different salts, glycerol and Triton X-100. A general overview of the stabilizing mechanisms (electrostatic, steric, and electrosteric) and the role of the different stabilizing additives has been investigated in detail with the use of $\mathrm{Al}_{2} \mathrm{O}_{3}$ as a model for ceramic samples. ${ }^{48}$ In slurry sampling ETV, the characteristics of the powders and the stability of the derived slurry are not as critical parameters as they are in slurry nebulization ICP techniques. The latter techniques require very finely dispersed suspensions to avoid clogging of nebulizers or sedimentation over time in the autosampler. Moreover, when introducing a slurry by means of a nebulizer into the ICP, size-distribution of the powder is critical, since very large particles might not be vaporized entirely in the ICP, leading to biased results. In slurry sampling ETV again, pre-vaporization of the sample is carried out in the furnace, and only a dry aerosol reaches the ICP. The ETV-generated aerosol consists of very fine particles which are produced during the re-condensation process in the cool transport line. Such aerosols are then easily vaporized and atomized or ionized in the ICP. Problems related to incomplete excitation of coarse particles in the ICP (as observed in slurry nebulization) are therefore avoided. Hence, in most of the recent papers discussing slurry sampling ETV, no additional grinding of the powder was carried out, and the samples were mostly used "as received" (see Table 2). Usually, after preparing the slurry suspension gravimetrically, the dispensing of the sub-aliquots is done volumetrically, thus allowing for much lower sample intake than in case of solid sampling ETV. This also allows measurement of several replicates from just one slurry, thus improving statistics. 


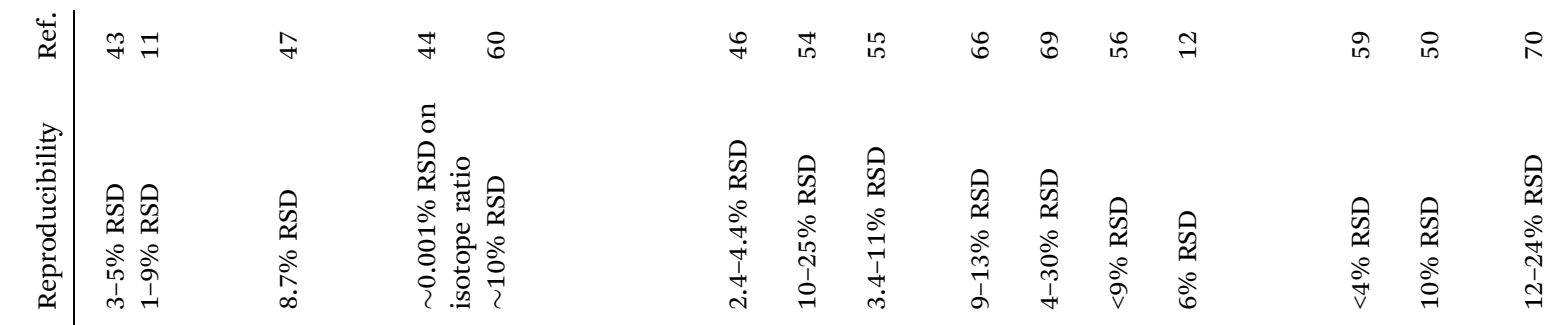

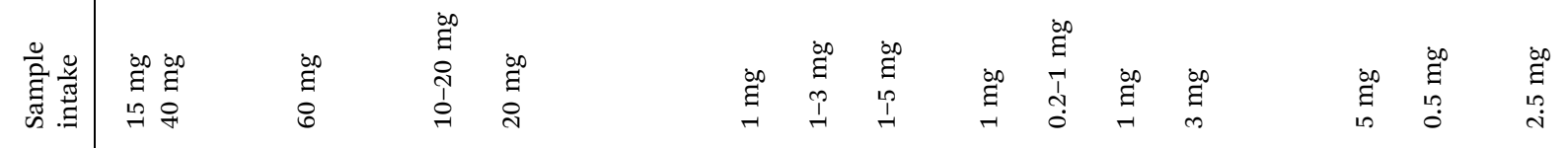

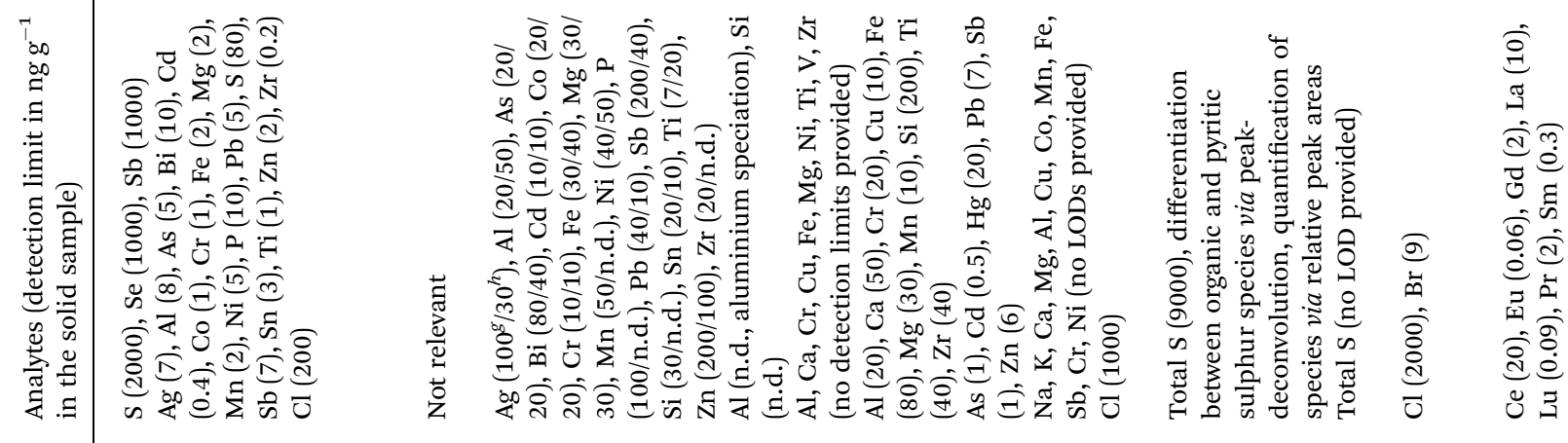
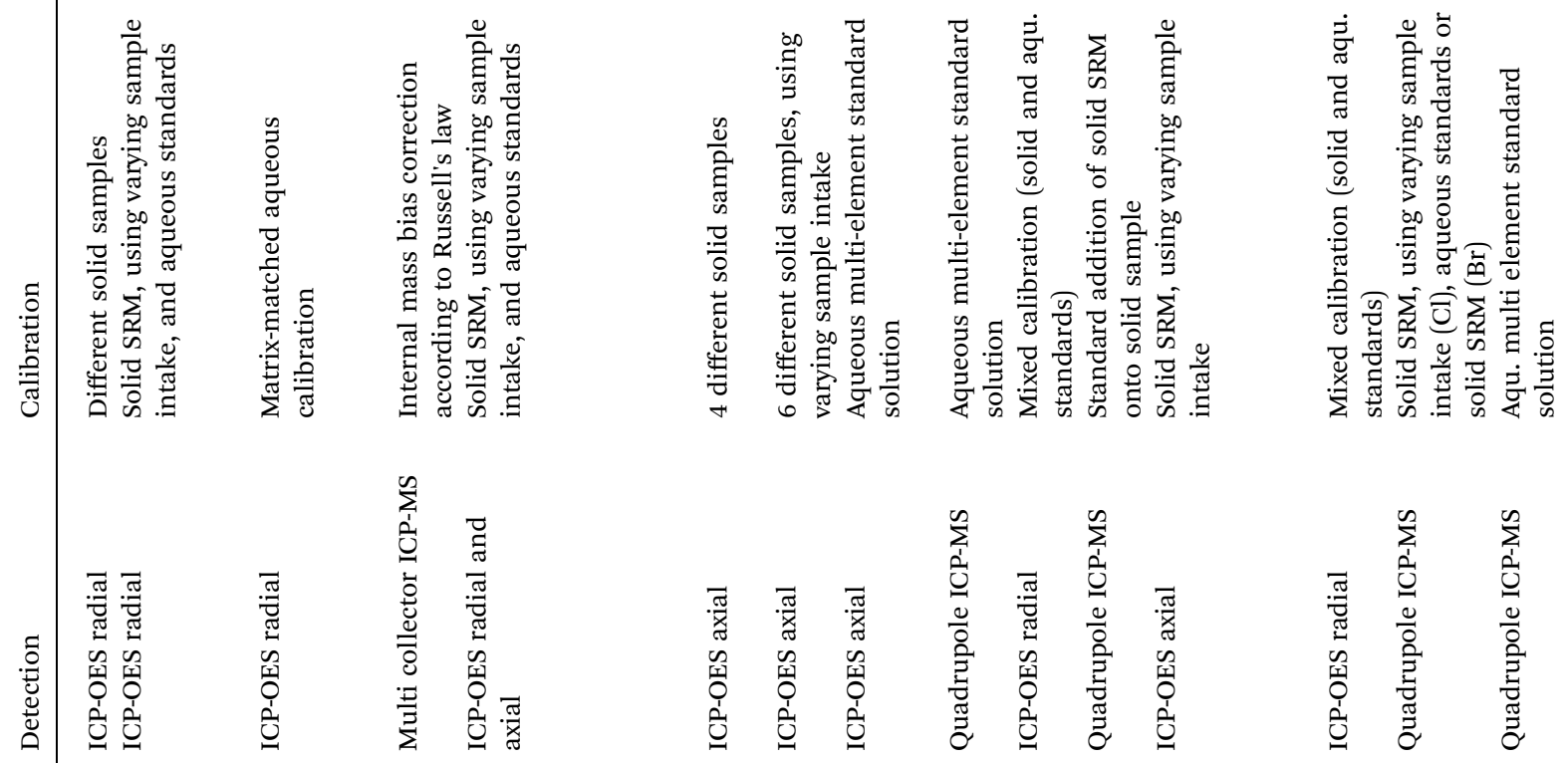

展
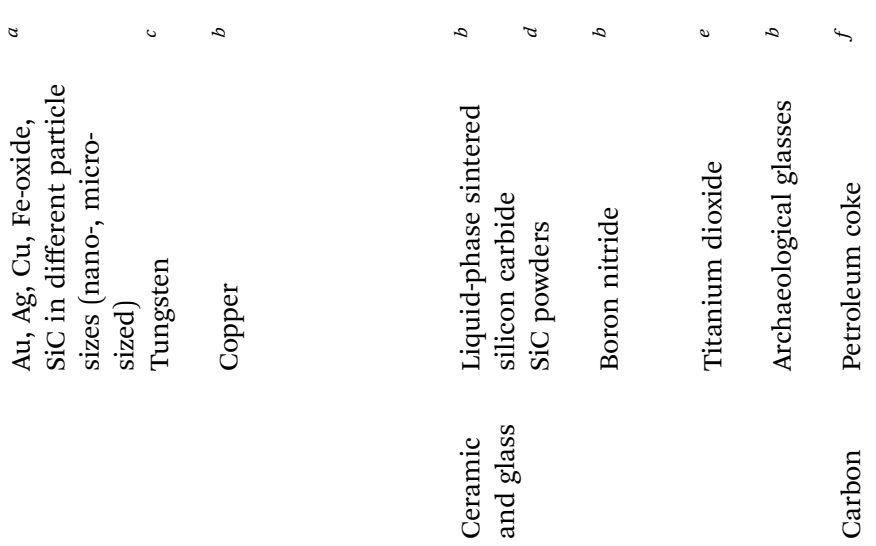


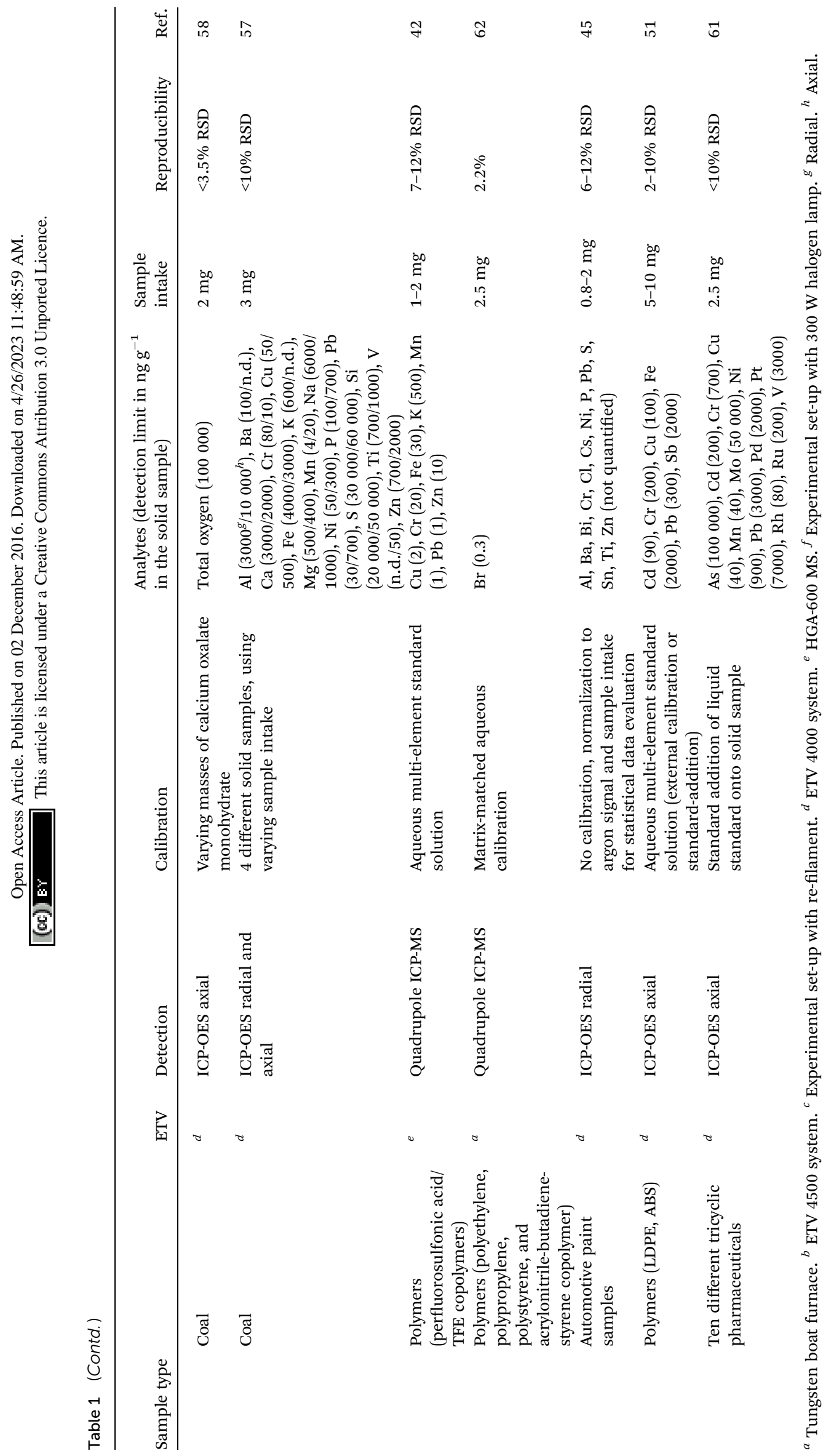


Typical sample intakes for slurry ETV are significantly below 1 $\mathrm{mg}$ (see Table 2).

\section{Signal quantitation, repeatability and detection limits}

Although quantitative measurements are desired in most cases, it has to be considered that quantification is not always necessary. Asfaw et $a l .^{45}$ have successfully discriminated between different types of red automotive paint chips, only by normalizing the signals to the sample intake and to one argon emission line. The temporal changes in the argon emission lines reflect the conditions in the ICP. Normalizing the intensities observed for target analytes to the argon signal allows therefore to account for temporal changes in plasma load. By covering variations in sample mass and plasma load, the authors were able to find significant differences in the elemental patterns of different car paints, and to distinguish between them using principal component analysis. Such a non-quantitative approach is very promising also for other applications which aim at source attribution (e.g., batch identification of raw materials, mineralogical fingerprinting via trace elements). The advantage of using such statistical approaches is that all type of variation can be used to achieve a discrimination between sample groups. This includes sample-dependent matrix effects, which therefore do not necessarily have to constitute an analytical "problem", as in case of fully quantitative analysis.

Regarding quantitation, there are two main approaches: (a) solid calibration and (b) liquid calibration. The first approach requires a material with identical (similar) matrix composition as the sample. The concentration of analyte in this material has to be known from an alternative analysis, or in case of a certified reference material from the certificate. Coverage of the expected analyte concentration range is another prerequisite for the calibration material. If such a reference material exists, results are very reliable, as matrix-effects are comparable for sample and standards. Unfortunately, such cases are rare due to the limited availability of appropriate reference materials. In order to adapt the working range, the following approaches are possible: (a1) vary the mass of reference material analyzed, in respect to the sample mass, or (a2) mix the reference material with other materials in order to obtain the desired analyte concentrations, or (a3) use a combination of varying sample intake and different reference materials. The first approach ignores matrix-effects which might increase with sample mass, the second assumes a perfect mixture of the reference standards, and the third assumes that matrix effects are constant between slightly different materials. All three are however very suitable approaches and are widely used - both in solid sampling ETV as well as in slurry sampling ETV (see Tables 1 and 2).

Alternatively, liquid standards can be brought into the graphite boats and dried. The dry residue can then be used for calibration. This approach can be done either by (b1) just adding an aqueous standard into an empty boat, or by (b2) adding the liquid standard onto dry sample material placed into the boat previously, or by (b3) adding the standard into the slurry before pipetting it into the graphite boat. Depending on the sample matrix, aqueous standards and spiked reference materials can give identical calibration curves ${ }^{49}$ for all analytes of interest, or just for a few. ${ }^{11}$ Even for one same matrix, varying behavior of different analytes is possible. ${ }^{50}$ Also slight variations in matrix (i.e., different types of polymers) can require the one or the other calibration approach..$^{51}$ This highly depends on the sample matrix and the analytes, and it is therefore often useful to check whether purely aqueous standards can be used, since this type of calibration is the most straight-forward one.

Apart from conventional calibration with liquid standards (external aqueous, external matrix-matched, standard addition), isotope dilution ICP-MS can also be applied in combination with ETV. ${ }^{52,53}$ However, this technique requires equilibration of isotopes prior to measurement, in order to guarantee identical behavior for the native isotopes and the spiked ones. Mixing liquid standards with solid samples might not be the ideal way of isotope equilibration. However, isotope dilution with ETV can have its merits, given the appropriate analytical question.

Sensitivity and reproducibility of ETV measurements are influenced to a large extent by the analyte-loading, i.e., the amount of sample introduced in the graphite boat. As mentioned previously, solid sampling ETV is typically performed with sample intakes in the range of several $\mathrm{mg}$ of dry sample. Contrarily, slurry sampling ETV allows dispensing much smaller sample masses. Commonly applied sample intakes in slurry sampling ETV are in the range of 0.006-0.2 mg (see Table 2). On the one hand, small sample intake can be advantageous in case of high analyte concentrations which might lead to detector saturation. On the other hand, Matschat et $a l .{ }^{54}$ and Barth et al..$^{55}$ have shown for ceramic functional materials that the repeatability improves with higher analyte concentrations. Therefore, adding the appropriate amount of sample into the ETV unit either by choosing solid sampling ETV (for higher intakes) or by choosing slurry sampling (for lower intakes) can be a way to bring a sample into the optimal working range of the applied detector, to optimize repeatability as well as sensitivity. Typical reproducibility of solid sampling ETV (often expressed as relative standard deviation of repeated measurement of one sample) ranges between $2 \%$ and $25 \%$ (see Tables 1 and 2). There is no apparent difference between solid sampling. ETV and slurry sampling ETV with regard to reproducibility. However, when compared to data obtained for sample digests analyzed using conventional liquid ICP-OES/MS reproducibility of ETV measurements is declined. This outcome can be explained with differences in the measurement principle, but it also reflects the influence of sample homogeneity, which is more important in case of ETV procedures than for digestion approaches with usually distinctly higher sample intakes. Nevertheless, limitations in reproducibility are compensated by the obvious advantages of solid-sampling, such as straightforward sample preparation, the absence of chemicals required for digestion, as well as a high sensitivity.

Detection limits are often expressed as instrumental detection limits, e.g., as absolute analyte masses that still can be detected. In the context of the analysis of advanced materials, it is however more interesting to compare detection limits which 
also consider the sample intake used. A very pronounced example which shows the difference between those two approaches of stating detection limits is the LOD reported for chlorine in different functional materials: Antes et al. ${ }^{56}$ and Gois et al. $^{50}$ report detection limits for chlorine of 1000 and 2000 $n g \mathrm{~g}^{-1}$ in petroleum coke and coal, respectively. Those values were obtained with ETV quadrupole ICP-MS. Nakata et al. ${ }^{\mathbf{4 7}}$ report a detection limit of $200 \mathrm{ng} \mathrm{g}^{-1}$ for chlorine in various nanoparticle powders, obtained by ETV-ICP-OES. Since ICP-MS is generally considered as the more sensitive technique, one might at first expect better detection limits than for ETV-ICPOES. But when considering the sample intake actually used (around $0.5 \mathrm{mg}$ in the two ICP-MS cases, and $60 \mathrm{mg}$ in the ICPOES case), the obtained LODs are put back into the expected proportion.

Generally, ICP-MS is the more sensitive method, compared to ICP-OES, also in combination with ETV. Especially for heavy elements, sensitivity is significantly better for ICP-MS than for ICP-OES, often for two to three orders of magnitude (see Table $1, \mathrm{Cd}, \mathrm{Pb}, \mathrm{Sb})$. This also holds true for elements which are difficult to analyze by means of ICP-OES, such as As. For elements in the medium mass range, ICP-MS is still better in terms of LOD, but with a difference of typically only one to two orders of magnitude (see Table $1, \mathrm{Fe}, \mathrm{Cu}, \mathrm{Zn}, \mathrm{Mn}, \mathrm{Cr}$ ). In specific cases, both methods can even be comparable in terms of LOD, for example when comparing the LOD of $\mathrm{Cr}$ in boron nitride $\left(20 \mathrm{ng} \mathrm{g}^{-1}\right.$, ICP-OES axial $){ }^{55}$ in coal $\left(10 \mathrm{ng} \mathrm{g}^{-1}\right.$, ICP-OES axial $){ }^{57}$ and in fluorinated polymers (20 ng g ${ }^{-1}$, quadrupole ICP-MS). ${ }^{42}$ All mentioned comparisons of LODs are obtained from comparable sample intakes in the range 1 to $5 \mathrm{mg}$.

ICP-OES can therefore perform quite similar to ICP-MS in terms of detection limits. This, and the fact that ICP-OES is an inherently more rugged technique might perhaps explain the fact that in the reviewed ten years there were about twice as many publications on solid sampling ETV using OES than using MS (see Table 1). However, in case of slurry sampling ETV, which typically works with 10 to 100 times lower sample intake, the superior sensitivity of ICP-MS is necessary. Consequently, most slurry-sampling ETV publications from the last ten years were done by means of ICP-MS (see Table 2).

\section{Application examples}

In the following paragraph, reports on methods developed for solid sampling and slurry sampling ETV in combination with ICP-techniques published in the last decade will be summarized. From the four groups of materials, three are covered by solid sampling ETV: metals and alloys, ceramics and glass, as well as carbon-based materials. In case of slurry sampling ETV coupled to ICP, only ceramics and glass, as well as carbon-based materials were investigated. As mentioned above, this might be related to the fact that it is not straight-forward to produce homogeneous powders from metal samples. Composite materials (the fourth group of samples discussed in this article) were not analyzed with ETV systems. This is perhaps due to the fact that such materials usually require analysis techniques with lateral resolution.
The analysis of coal and related products has been mostly studied using direct ETV approaches - out of 8 publications in the period 2005 to 2016 only one used slurry-sampling. Interestingly, five of the most recent publications in this field study rather unconventional analytes. For example, the quantification of halogens (chlorine, bromine) in coal was studied, ${ }^{50,56}$ as well as total oxygen content ${ }^{58}$ (a parameter conventionally not accessible via ICP techniques), and sulphur. ${ }^{12,59}$ In case of metallic samples, not only trace impurities were analyzed, ${ }^{\mathbf{1 1 , 6 0}}$ but also chlorine, ${ }^{47}$ as well as the isotopic composition of the matrix metal tungsten. ${ }^{44}$ Synthetic organic materials such as polymers and pharmaceutical components were analyzed for the presence of trace elements ${ }^{42,51,61}$ as well as for bromine. ${ }^{62}$

All of the mentioned sample types have in common that they are difficult to dissolve, hence the choice of ETV. In most of the cases of slurry sampling ETV, the powders were analyzed as received. This avoids possible contamination, and as mentioned above, powder characteristics are not very stringent in case of ETV, since the sample is vaporized in two stages (first, in the ETV, and then once more in the ICP). The suspending medium varies from pure water to complex mixtures and has to be optimized on a case-to-case basis. Reported detection limits, expressed as concentration in the solid sample (see Table 1), obtained from ETV slurry measurements are typically comparable with those obtained from conventional liquid analysis using the same ICP detector. Xiang et al. ${ }^{63}$ performed a study comparing slurry nebulization and slurry-sampling ETV in which they found that in the case of titanium dioxide powder samples, the relative sensitivities of both methods are comparable. However, the absolute sensitivity was better for slurrysampling ETV. This is due to two factors: firstly, as opposed to wet-chemical digestion, only a minor dilution of the solid sample occurs. As mentioned in a review by Wang and Yang, ${ }^{23}$ in some cases suspensions of up to $30 \%(\mathrm{w} / \mathrm{v})$ can be analysed via ETV slurry analysis, whereas chemical digestion typically yields solutions of less than $1 \%(\mathrm{w} / \mathrm{v})$. Secondly, the sample introduction efficiency is around 10 to $20 \%$ with ETV, as investigated by Peschel et al. in a study using alumina and radioactive tracers. ${ }^{64}$ Slightly increased values ranging from 26 to $50 \%$ were reported by Ertas and Holcombe, ${ }^{65}$ indicating that further improvements are possible with the use of $\mathrm{Pd}$ or $\mathrm{NaCl}$ as carrier. Compared to those values, nebulizers have by far a worse sample introduction efficiency (typically around 1-5\%). Although better sample introduction efficiency is advantageous in terms of sensitivity, it comes at the cost of a high matrix-load. However, it is possible to adjust the temperature program of the graphite furnace in such a way, that matrix and analyte are introduced at different times to the ICP, thus alleviating the problem of high plasma load. ${ }^{66}$ This is true for both solidsampling ETV and slurry-sampling ETV.

\section{Temporal separation of sample constituents with different volatility}

In contrast to traditional liquid ICP-OES or ICP-MS analysis, but also other solid-sampling techniques such as laser ablation ICP or slurry nebulization ICP, sample introduction via ETV offers 
the possibility to separate analyte and matrix prior to measurement. Whereas in the former approaches all sample constituents are introduced into the plasma at the same time, ETV allows a separation of volatile from less volatile sample constituents. This unique property of ETV can be used to overcome spectral interferences in ICP-OES or ICP-MS analysis, but also to differentiate between elemental species with different vaporization behavior (elemental speciation).

In many cases, the vaporization behavior of the sample matrix and of the analyte is different. Thus, with the selection of a proper temperature program the release of analyte and matrix can be separated in time. If the matrix is more volatile than the analyte, this allows purging the vapors generated by the matrix directly into the exhaust. ${ }^{42}$ In this case only the analyte vapors are brought to the ICP. Discarding the pyrolysis products of the matrix is advantageous in case of end-on ICP systems (i.e., ICPMS or axial view ICP-OES), since contamination of cones or windows is held at bay. However, the ETV system must be equipped with suitable switches to re-direct the gas stream, and the only commercial system available today (ETV 4000, Spectral Systems) does not offer this feature. Yet, the following examples will show that even without a bypassing option, thermal resolution is an interesting feature.

Separating analyte and matrix in time is a powerful feature of ETV, also in cases where the analyte is released earlier than the matrix. Hassler and coworkers ${ }^{\mathbf{1 1}}$ have recently developed a method to selectively release trace element impurities from molten copper droplets in the presence of gaseous fluorination or hydrogenation modifiers. While the copper mostly remains in the graphite furnace, the analytes are quantitatively transported to the ICP-OES detector. The advantage of such partial vaporization is that copper-deposition in the transfer line between ETV and ICP is reduced, and that non-spectral matrixeffects due to copper in the ICP are minimized. Aramendía and coworkers $^{66}$ have used thermal separation to tackle a more specific challenge. When analyzing $\mathrm{Zn}$ in $\mathrm{TiO}_{2}$ ceramic via ETV quadrupole ICP-MS, spectral overlap of ${ }^{50} \mathrm{Ti}^{16} \mathrm{O}^{+}$on ${ }^{66} \mathrm{Zn}^{+}$ hinders correct quantification. However, when analyzing the analyte at a temperature where the matrix is not yet vaporized, this problem can be readily circumvented. A similar observation was made by Lin et al.,${ }^{41}$ when analyzing ${ }^{111} \mathrm{Cd}^{+}$with quadrupole ICP-MS. The ammonia reaction gas used to remove spectral interferences on another analyte (chromium) was found to produce the adduct ion ${ }^{94} \mathrm{Mo}^{14} \mathrm{NH}_{3}{ }^{+}$in the presence of molybdenum. This results in spectral overlap at mass 111, and gives erroneous results for cadmium. However, since $\mathrm{Cd}$ and Mo vaporize at different temperatures (and therefore at different times during the ETV program), a separation of the two signals at mass 111 was possible.

Vaporization behavior can also be used to determine different species of one single element. ${ }^{67}$ As demonstrated by Gelaude et al. ${ }^{68}$ it is possible to distinguish inorganic mercury from methyl-mercury in organic samples by means of ETV ICPMS, because the two groups of $\mathrm{Hg}$-species evaporate at different temperatures. In the field of functional materials, there have only been few examples of this technique. Hassler et al. ${ }^{\mathbf{4 6}}$ investigated liquid-phase sintered silicon carbide. This material contains aluminum in the binder-phase, as well as aluminum which is incorporated into the SiC grains during the sintering process. As the properties of the material depend on the amount of aluminum inside the SiC grains, an ETV method was developed to distinguish between aluminum in the binder phase and aluminum in the SiC grain boundary. By applying a slow heating ramp in the ETV system, the authors demonstrated that it is possible to distinguish between those two aluminum-containing phases and to obtain quantitative information about the phases, apart from the mere total aluminum content of the sample which is obtained after digesting the sample. Bauer et al. ${ }^{12}$ showed that different sulphur species present in coal can be effectively distinguished from one another, since they exhibit different volatility. This can help in choosing appropriate coal treatment strategies to reduce the concentration of sulphur in the coal prior to combustion (the sulphur-reduced coal can then be considered as "functional" raw material, as its initial properties were changed to obtain better performance).

To summarize, the correct temperature program in ETV helps in overcoming non-spectral matrix-effects by removing the matrix prior to analysis, but it can also be used in a more elegant way by separating analyte and parent-elements of spectral interferences in time. Moreover, different groups of analyte species can be distinguished from one another, giving access to additional information with regard to the chemical form of the analyte.

\section{Limitations and future possibilities of ETV sampling coupled to ICP-based detection techniques}

As summarized in this chapter, ETV has been used for various applications from the field of functional materials throughout the last years. One of its key features is the fact that samples can be analyzed without often troublesome chemical digestion. Thus, sample throughput can be increased distinctly, which is essential for routine applications. But more importantly, sensitivity of analysis could be improved since undiluted samples are measured. Although, it has to be mentioned that the introduction of more sample material into the ICP can negatively affect quality of measurement. Another benefit of ETV for analysis of advanced materials is the variety of available options for signal quantification. Besides the use of certified reference materials it is also possible to apply the concept of standard addition; under certain circumstances even calibration with aqueous standards is feasible.

Conventional quantitative measurements with ETV in combination with ICP-based techniques will remain a valuable tool in the context of industrial measurements (e.g. qualitycontrol), while method development in the scientific context can focus on more advanced applications such as temperaturedependent fractionation to resolve spectral interferences, or to determine analyte-speciation, as well as non-quantitative approaches for the purpose of fingerprinting in forensic applications. However, although ETV in combination with ICP-MS/ OES detection has become an established analytical technique, the use in routine laboratories is still limited. To further 
support the acceptance of ETV for analysis of functional materials methodical improvements are required. In particular full spectra acquisition is needed - for ICP-OES as well as for ICP-MS a prerequisite for accurate correction of background changes during an ETV cycle. Thus, besides removal of spectral interferences also the number of simultaneously measured elements could be increased. Furthermore, fully automated signal processing is desired, making evaluation of transient ETV signals easier and thus more user friendly.

\section{Methods employing LA-ICP-MS and LA-ICP-OES in material science}

Laser Ablation (LA) uses a focused laser beam to remove material from a solid sample surface. Coupled to an elemental detection technique such as ICP-MS or ICP-OES, LA can be used for chemical analysis. During irradiation of the sample surface with a pulsed laser beam, a dry sample aerosol is generated. This aerosol is transferred from the sample chamber to the ICP using an inert carrier gas. Here, the generated aerosol particles are vaporized, atomized, and ionized to be transferred into a high vacuum for subsequent detection of the ions in a mass spectrometric assembly (ICP-MS). Alternatively, element specific radiation emitted by excited analyte atoms/ions can be collected and analyzed by means of an ICP-OES, omitting the step of transition into a high vacuum. Detailed information about the used instrumentation and the ablation process can be found elsewhere. ${ }^{21,75,76}$ Nowadays, LA-ICP-MS is a well-established method for the micro-local analysis of various sample types. Notable applications are found in the geosciences and the life sciences. Besides, LA-ICP-MS has also been used for the analysis of technologically relevant samples and materials. Key features of this technique are the possibility of laterally resolved analysis with achievable resolutions in the low $\mu \mathrm{m}$-range, as well as high sensitivity offering detection limits in the $\mathrm{ng}^{-{ }^{-1}}$. scale. Especially important for material analysis is the opportunity of depth profile analysis, where the achieved depth resolution depends on the used instrumentation and/or the analyzed material. The low need of sample preparation is a large benefit, especially when high-purity materials need to be analyzed; the risk of sample contamination can be kept at a minimum. As in most other solid sampling techniques, one important aspect of LA-ICP-MS is signal quantification. ${ }^{77}$ The ablation process as well as the ionization of the generated aerosol in the ICP are highly matrix-dependent. Thus, standards with a composition that is closely matched to the samples have to be used. Otherwise, reliable quantification cannot be guaranteed. If certified reference materials for the analyzed sample types are available, those can readily be used for calibration and signal quantification. For many materials, however, no certified reference materials are available. In this case, suitable standard materials have to be prepared in-house. It is generally accepted that matrix dependence of the ablation process decreases with lower laser wavelengths. The same statement is true for decreasing pulse width from the ns into the fs range. However, by decreasing pulse width and wavelength of irradiation, the price of the device will rise. Thus, a tradeoff is in most cases considered being appropriate. For example, $213 \mathrm{~nm}$ frequency quintupled Nd:YAG lasers are widely distributed in LA laboratories. They offer weighted ablation characteristics paired with a reasonable price. Especially when depth profiling applications are of interest, $193 \mathrm{~nm} \mathrm{ArF*} \mathrm{excimer} \mathrm{lasers} \mathrm{can} \mathrm{be} \mathrm{a} \mathrm{preferable}$ option. Such instruments offer high homogeneity of the energy distribution in the laser beam and thus lead to the formation of almost perfectly cylindrically shaped ablation craters in most materials - a prime prerequisite to avoid blurring of depth profiles. Detailed discussions of the state of the art using LAICP-MS in materials science will be given in the following section.

\section{LA-ICP-MS/OES for bulk analysis}

Bulk analysis using LA-ICP-MS and LA-ICP-OES represents the simplest type of analysis: it requires only minimal data treatment and provides information about average concentrations of the investigated analytes in the sample. A major aspect that has to be considered is that the analyzed area needs to be representative for the bulk composition of the sample in order not to distort the obtained results. Typically, larger areas of interest are ablated (line-scan patterns or multi-spot patterns). In the field of advanced materials, LA-ICP-MS/OES has been used for bulk analysis of two main sample types: polymer samples and steel samples.

Properties of polymers may strongly depend on the concentrations of inorganic additives in the material. Reliable quantitative analysis of such compounds is imperative for quality control, as well as for safety reasons. For example, legislation of the European Union regulates the amounts of heavy metals permitted in polymers. Liquid ICP-OES and ICP-MS are commonly used for trace metal determination in such samples. ${ }^{78}$ However, sample preparation includes timeconsuming digestion steps and the risk of contaminations is rather high. Therefore, direct solid sampling methods are an attractive alternative. One of the most widely used methods is X-ray fluorescence (XRF). However, sensitivity is often not sufficient especially for lighter elements. Therefore, LA-ICP-MS is considered a possible alternative, which requires no or only minor sample pretreatment and offers excellent sensitivity. Because of these obvious advantages, the use of LA-ICP-MS for polymer analysis has been proposed already in the 1990s..$^{79,80}$ The most problematic aspect in all these early works was quantification: suitable certified reference materials were not available and thus, in-house standards had to be prepared, which significantly increased workload and therefore hampered attractiveness of the method. In later years, the use of improved ICP-MS instrumentation (e.g., collision/reaction cell technology ${ }^{81}$ and the manufacturing of certified reference materials has considerably broadened the application range of LA-ICP-MS for polymer analysis. Nowadays, at least some certified reference materials composed of different polymers are available, provided for example by BAM (Bundesanstalt fuer Materialforschung und-pruefung, BAM-H010, matrix: acrylonitrilebutadiene-styrene terpolymer) or IRMM (Institute for Reference 
Materials and Measurements, ERM-EC 680 and 681, matrix: polyethylene). Besides methodical improvements, some industrially relevant applications such as the analysis of bromine and tin, ${ }^{82}$ as well as the analysis of waste polymers ${ }^{83}$ have been presented in the past years. Some recently presented applications deal with distribution analysis of trace elements in polymers and will be discussed in a later part of this review.

In steel industry, the assessment of trace element concentrations is equally important as in polymer science. Besides the contents of major elements and the production process, mechanical properties of steels may also depend on the occurrence of minor and trace elements. ${ }^{84}$ In analogy to polymer samples, commonly used methods for steel analysis are acid digestion of the samples and subsequent ICP-MS or ICPOES measurement, whereby the already mentioned drawbacks arise. Due to the large number of available certified reference materials, quantification of major, minor, and trace elements in steel is not considered a problem, even if most of those standards are not certified for (and lack in) their microhomogeneity. Due to rather simple quantification, the number of publications presented for method developments in the field of bulk investigations is rather low. Already in the early 1990s, LA-ICP-MS has been successfully used for steel analysis. ${ }^{85}$ Only a couple of further reported works can be found in literature. Compared to investigations with nanosecond laser instrumentation, ${ }^{\mathbf{8}}$ Wiltsche and Günther performed LA-ICP-MS experiments with femtosecond lasers, coming to the conclusion that calibration even without matrix-matched standards is feasible.$^{87}$ A comparison between ns-LA-ICP-MS and fs-LA-ICPMS for the analysis of boron in steel has been presented by Kurta et $a l .{ }^{88}$

Besides the analysis of polymers and steels using LA-ICP-MS/ OES, also some applications for bulk analysis of materials used in semiconductor industry have been reported. Khvostikov et $a .^{89}$ successfully quantified trace elements in lanthanum gallium silicate by LA-ICP-MS, Lee et al. ${ }^{\mathbf{9 0}}$ investigated the composition of $\mathrm{Cu}(\mathrm{In}, \mathrm{Ga}) \mathrm{Se}_{2}$ thin films used for the production of solar cells. Cakara et al. ${ }^{91}$ recently presented a multi-variate calibration method for the analysis of Mo-Si-B alloys used as oxidation resistant coating.

\section{Laser ablation in liquid (LAL) with subsequent slurry analysis}

As mentioned in the previous chapter, the use of an appropriate reference material is mandatory for accurate LA-ICP-MS analysis. Availability of suitable certified reference materials - in terms of composition, but also covered elements and contents is therefore a prerequisite for the acceptance of LA-ICP-MS as an alternative to traditional procedures for the quantitative analysis of advanced materials. Recently, a new approach has been proposed to overcome the need of CRMs. The principle of this new technique is based on the transformation of the compact solid sample into colloidal solutions of nanosized analyte particles by means of laser ablation. Subsequent analysis of the prepared slurry provides quantitative information about the composition of the initial sample. ${ }^{92}$ This so called "laser ablation in liquid" (LAL) technique has been widely used for the industrial production of nanoparticles with controlled dimensions and the ablation mechanisms have been extensively studied. ${ }^{93,94}$ For analytical purposes, the material ejected from the sample surface is trapped in the liquid medium in the form of a suspension or as dissolved ions. Finally, the derived solutions are analyzed for the elements of interest using conventional ICP-OES or ICP-MS instrumentation with liquid sample introduction.

For ablation, the sample is immersed in a liquid medium and the laser beam is delivered to the sample surface. Laser instrumentation applied so far include Nd:YAG laser systems, operated at the fundamental wavelength of $1064 \mathrm{~nm}$ (ref. 92) but also frequency tripled $(355 \mathrm{~nm})^{95}$ and quintupled $(213 \mathrm{~nm})^{96}$ systems, as well as a titan-sapphire femtosecond laser system (wavelength $780 \mathrm{~nm}$ ). ${ }^{97}$ Typical ablation parameters for systems with pulse duration in the ns range are repetition rates of 1$20 \mathrm{~Hz}$, with laser energies varying from $0.50 \mathrm{~mJ}$ to $100 \mathrm{~mJ}$. Beam diameters ranged from tens of $\mu \mathrm{m}$ to some hundred $\mu \mathrm{m}$ resulting in laser fluencies in the order of $<10-480 \mathrm{~J} \mathrm{~cm}^{-2}$. With fs-instrumentation higher repetition rates $(250$ and $500 \mathrm{~Hz}$ ) with a constant fluence of $20 \mathrm{~J} \mathrm{~cm}^{-2}$ have been used. In all LAL applications until now, the use of high purity water as liquid medium is reported. Properties which favor the use of water are high transmission in the wavelength-range of the applied laser systems, the low vapor pressure and the availability with required purity. Depending on the task, the depth of the water layer above the sample surface varied between $2 \mathrm{~mm}$ and $30 \mathrm{~cm}$; increased liquid volumes result in a less concentrated solution but allow reducing the risk of material losses due to nanoparticle ejection caused by the shockwave induced by laser ablation. After the ablation process, the sample is separated from the liquid and rinsed with water to remove nanoparticles remaining on the surface. For chemical analysis, sample solution and rinse solution are combined, and measured using standard procedures. Due to the small particle sizes (depending on the applied laser parameters, but usually below $100 \mathrm{~nm}$ ) the particle suspensions reveal no differences to conventional liquid samples during ICP-OES or ICP-MS measurement, thus analyte concentrations in the investigated sample solutions can be assessed using traditional calibration functions determined with aqueous standards. Based on the derived results, analyte contents or the composition of the original solid can be determined, either by using a sample inherent internal standard, or by measuring the volume of the ablated material.

The first application of LAL has been reported for the analysis of the composition of zinc-aluminum-copper alloys. Derived results were found to be in good agreement with reference values, indicating the applicability of this new method for analysis of major and minor alloy constituents. ${ }^{92}$ Since such alloys are easy to dissolve, the advantages of LAL compared to standard approaches are fast sample pretreatment and especially the practically non-destructive analysis - which is attractive for the analysis of valuable samples. A more challenging task is the analysis of the $\mathrm{Al} / \mathrm{Na}$ ratio in cryolite $\left(\mathrm{Na}_{3} \mathrm{AlF}_{6}\right)$, a material used for the reduction of alumina $\left(\mathrm{Al}_{2} \mathrm{O}_{3}\right)$ to metallic aluminum by the Hall-Herault process. Conventional AAS or ICP-OES analysis requires dissolution of the test specimen prior 
to analysis. Due to the refractive nature of cryolite, this sample pretreatment step is rather time-consuming and demands expensive equipment. By combining liquid phase laser ablation with ICP-OES and total reflection X-ray fluorescence analysis of the derived colloidal solution, an adequate determination of $\mathrm{Al} / \mathrm{Na}$ ratios was possible, indicating that this technique could be used for industrial diagnostics of electrochemical cells. ${ }^{95}$ In a recent study, the particle size-related elemental fractionation during LAL sampling of NIST SRM 610 (National Institute of Standard and Technology, Maryland, USA) was evaluated. ${ }^{\mathbf{9 8}}$ Particles generated by LAL were divided into two size classes (below and above $0.4 \mu \mathrm{m}$ diameter) by filtration, and subsequently analysed using ICP-MS. Comparison between the results obtained by slurry nebulization of LAL solutions and those obtained after acid digestion of the same colloidal sample solutions revealed an enrichment of volatile elements (e.g., As) in the smaller particles and depletion in the larger particles. Information about the occurrence of elemental fractionation in nanoparticles smaller than $0.1 \mu \mathrm{m}$ - as observed in other LAL studies $^{92,97}$ - is not reported.

Beside measurement of the average sample composition, the LAL approach has also been used for the analysis of isotopic ratios. Okabayashi et al. investigated the capability of LAL for isotopic analysis of solid samples in detail, including elemental and isotopic fractionation effects during LAL sampling. ${ }^{97}$ The data obtained from the analysis of two standard reference materials, NIST SRM 610 glass and the IRMM-014 high-purity Fe metal (Institute for Reference Materials and Measurements, Geel, Belgium), demonstrated that after careful optimization of laser ablation conditions, the LAL technique can be applied for isotopic analyses. For example the ratios obtained for ${ }^{238} \mathrm{U} /{ }^{232} \mathrm{Th},{ }^{208} \mathrm{~Pb} /{ }^{206} \mathrm{~Pb},{ }^{56} \mathrm{Fe} /{ }^{54} \mathrm{Fe}$ or ${ }^{57} \mathrm{Fe} /{ }^{54} \mathrm{Fe}$ ratios did not vary significantly $(<0.5 \%)$ from the reference values.

In a further application of the LAL technique, a freestanding droplet that acts as a micro-laser cavity ${ }^{96}$ has been used to avoid immersion of the whole sample in the liquid medium. Thereby, an improvement in sensitivity could be achieved, since more concentrated solutions could be prepared. Furthermore with this approach the use of commercial LA systems with minimal or no modification is enabled. Applicability for the analysis of glass samples has been demonstrated by measurement of NIST SRM 611. For sample ablation with a $213 \mathrm{~nm}$ system, a droplet with a volume of $25 \mu \mathrm{L}$ was deposited on the sample surface. After firing 60.000 shots with a laser energy of $0.084 \mathrm{~mJ}$ and a spot size of $34.3 \mu \mathrm{m}$, the resulting solution was collected by means of a pipette and transferred into a centrifuge tube. Concentrations derived via ICP-MS analyses using aqueous calibration were in good agreement with the certified values, measurements of the uranium/thorium ratio were very close to the expected value.

Summing up, LAL allows the analysis of materials that are insoluble or only soluble in solvents that are incompatible with the ICP. Compared to other techniques for the analysis of solid samples issues related to signal quantification are of only minor importance, since prepared particle suspensions are uniform, stable (no sedimentation problems) and behave very similar to clear solutions. Another benefit is the possibility of sample accumulation through laser ablation with multiple shots and/or spots, thus sensitivity of measurement can be easily adjusted.

\section{Depth profiling: LA-ICP-MS/OES for the analysis of layered samples}

Especially in the semiconductor industry and the engineering of protective coatings, layered samples are widely used. The chemical analysis of such systems is often considered as a major challenge: the actual composition, trace impurities, or film homogeneity and thickness might be only some aspects that need to be investigated. Compared to other typical methods applied for depth profiling, such as time-of-flightsecondary ion mass spectrometry (ToF-SIMS) or other ionprobing techniques, ${ }^{99}$ LA-ICP-MS offers weaker depth resolution. This, however, gives the opportunity to analyze depths of several $\mu \mathrm{m}$ until hundreds of $\mu \mathrm{m}$ in a reasonable amount of time, which is not possible using afore-mentioned techniques. Additionally, the use of LA-ICP-MS offers the possibility to analyze conducting as well as non-conducting samples. No vacuum is required, which is of minor importance in case of technological materials, but is advantageous for other sample types (e.g. biological materials). In contrast, GD-MS/OES offers similar characteristics to LA-ICP-MS, however, providing significantly lower lateral resolution and having rather fixed requirements regarding the sample geometry. Furthermore, sample throughput in LA-ICP-MS is considerably higher.

If the goal is to study the in-depth distribution of analytes using LA-ICP-MS, current problems are mostly related to the characteristics of the used laser beam. Even if major improvements have been made during the last decades, still some drawbacks remain. The use of UV and deep-UV lasers enhances the ablation behavior of the samples and Gaussian beam profiles can be converted into flat-top profiles by the application of beam homogenizers, which dramatically increases depth resolution. ${ }^{\mathbf{1 0 0}}$ Femtosecond (fs) instrumentation also leads to improved crater profiles and additionally provides lower ablation rates paired with minimal elemental fractionation compared to nanosecond lasers. Mateo et al. ${ }^{13}$ demonstrated that even for complex layer systems (polymer coated steel samples) fs-LA-ICP-MS can offer depth profiling capabilities comparable to GD-OES, but with considerably higher lateral resolution. If material from the crater walls is sampled, signalcarryover between sample layers will occur, causing blurred depth profiles. Especially for the cheaper and thus more widely distributed nanosecond laser systems, depth resolution of the LA-ICP-MS measurements is impaired by conical crater profiles. Nevertheless, a number of applications has presented in the last years. Gutiérrez-González et al. ${ }^{\mathbf{1 0 1}}$ successfully analyzed photovoltaic devices containing CdTe using LA-ICP-MS. Comparison with GD-ToF-MS and SIMS yielded good agreement between the obtained depth profiles. In this work, a $193 \mathrm{~nm} \mathrm{ArF} *$ flat-top profile laser system was used, which seems to be very suitable for depth profiling applications. Similarly equipped LA-ICP-MS systems were used by Balcaen et al. ${ }^{\mathbf{1 0 2}}$ for the analysis of layered high-tech materials. Bleiner et al. ${ }^{\mathbf{1 0 3}}$ used a similar laser system in combination with ICP-ToF-MS for the analysis of Ti-based 
coatings. Hrdlička et al. ${ }^{104}$ employed LA-ICP-OES for the analysis of $\mathrm{Zn}$-based coatings. Also lasers with longer wavelengths have been used: for example, Lee and $\mathrm{Lim}^{\mathbf{1 0 5}}$ reported on the analysis of TiN layers using a $213 \mathrm{~nm}$ ns-LA-ICP-MS system, concluding that cooling of the sample surface between consecutive laser shots (i.e., firing the laser at low repetition rates) would also permit good depth profiling capabilities of such LA-ICP-MS systems. In combination with ICP-OES, lasers with varying wavelengths showed to offer excellent depth profiling capabilities as demonstrated by Kanicky et al. for tungsten carbide ${ }^{106}$ and metal-zirconia ceramic layers ${ }^{107}(266 \mathrm{~nm}$ Nd:YAG) as well as Zr- and Ti-based nitride layers ${ }^{108}$ (355 nm Nd:YAG). Zaoralkova et al. ${ }^{109}$ reported depth profiling using $1064 \mathrm{~nm}$ Nd:YAG instrumentation alongside with LA-ICP-MS linescan measurements on layered silicate ceramics. An application oriented question has been presented by Michalska et al: ${ }^{110}$ they investigated the stability of ion-selective electrodes by LA-ICP-MS depth profiling. A special application has been presented by Hattendorf $e t$ al.; in the reported work, LA-ICP-MS was used for the determination of layer thicknesses in the single-digit nmscale and below. ${ }^{14}$ To achieve this task, standards with known layer thickness were used for signal quantification.

An alternative for depth profiling omitting the use of LA instrumentation has been recently presented by Rupp et al. employing ICP-OES, ${ }^{111}$ as well as ICP-MS detection: ${ }^{112}$ they used a dynamic etching protocol with element specific on-line detection to study the stoichiometry of surface-near regions of complex metal oxide thin films. This approach offers two distinct advantages compared to LA analysis; aqueous standard solutions can be used for quantification and a differentiation of species with varying solubilities can be obtained by subsequent application of different leaching agents.

\section{Laterally resolved analysis using LA-ICP-MS}

Undoubtedly, one strength of LA-ICP-MS is the possibility of performing laterally resolved analyses with resolutions until the low $\mu \mathrm{m}$-scale. The achievable lateral resolution is mainly depending on the size of the focused laser beam and can be adjusted according to sample-specific requirements. Regarding high lateral resolutions, a couple of improvements have been made during the last years. Ultra-fast washout cells ${ }^{113}$ and even mathematic data treatment approaches such as deconvolution ${ }^{114}$ have led to the possibility of performing distribution analysis at the sub- $\mu \mathrm{m}$ scale. This opportunity might also be interesting in some applications for advanced materials. Even if element mapping using LA-ICP-MS is widespread in the life sciences and in geology, this method has not been completely exploited for the analysis of technological samples. Some of the already mentioned advantages would make LA-ICP-MS perfectly suitable for many applications. The possibility of fast elemental mapping of polymers has for example been described by Dobney et al. ${ }^{115}$ This group proposed an LA-ICP-MS method as an alternative for the rather time-consuming elemental mapping using solid sampling-Zeeman atomic absorption spectrometry (SS-ZAAS), cutting a total analysis time of 5-6 hours down to 1520 minutes. Additionally, it needs to be considered that SS-ZAAS is a single-element technique, whereas by LA-ICP-MS multiple elements can be analyzed within one cycle of analysis. In the field of semiconductor industry, Stika et al. ${ }^{116}$ used LA-ICP-MS in combination with ToF-SIMS for the analysis of photovoltaic modules and a study of their degradation. Zoriy et al. ${ }^{117}$ mapped elemental distributions on microelectronic devices using LAICP-MS. The imaging capabilities of LA-ICP-MS have been also used for metal analysis. Latkoczy et al. ${ }^{118}$ investigated element distributions in $\mathrm{Mg}$ alloys and compared the results to EPMA analysis with the conclusion that LA-ICP-MS analysis is superior in the assessment of trace elements. Hu et al. ${ }^{119}$ investigated the distributions of trace elements in Ni alloys and found segregation of certain elements within the grain structure. Gierl-Mayer et al. ${ }^{120}$ presented a study on the distribution of boron in powder metallurgically produced steel samples by LA-ICP-MS which showed to be accumulated on the grain boundaries. Besides lateral distribution analysis, they also quantitatively investigated the depth profile of boron caused by the sintering process, indicating depletion of boron in the outermost sample layers. A medically related study of materials has been recently presented by Draxler et al.;21 they investigated the distribution of $\mathrm{Mg}$ in bones after implantation of prostheses made from Mg-alloys.

\section{Limitations and future possibilities of LA sampling coupled to ICP-based detection techniques}

As demonstrated in this chapter, the applications for LA-ICP-MS in the field of material science are various; in Table 3 the literature discussed within this article is summarized. However, compared to other fields of science utilizing LA-ICP-MS, still not all possibilities have been exploited in material science. For instance, in the field of biosciences, LA-ICP-MS mapping is very often used for trace element mapping in different tissue types and has become a popular technique during the last years. As a sensitive and rapid method, it would also be very suitable for mapping of trace elements in technological samples, such as metals or ceramics. When investigating such samples, not only the bulk composition might be important; for example the grain structure could be correlated with measured elemental distributions that are not accessible by typically applied methods like EPMA, due to their concentration and/or atomic weight (e.g., boron, as demonstrated for bulk analysis by Kurta et al. ${ }^{88}$ ). Recent advances and developments in laser technology also helped to improve the versatility of the method. Deep-UV-ns or fs instrumentation offers outstanding properties regarding the achievable beam profiles and also simplify quantification even without the use of matrix-matched standards. Additionally, due to almost no restrictions on sample size and physical properties (e.g., conductivity), the method is very versatile and not restricted to the analysis of one sample type. Thus, metallic samples could be analyzed alongside with polymers and ceramics in one instrument without changing the instrumental setup or the sample preparation strategy. Even the analysis of special samples where different material properties are combined, as demonstrated by Mateo et al. for the analysis of polymer-coated steel samples, ${ }^{13}$ becomes feasible. 
嵌|

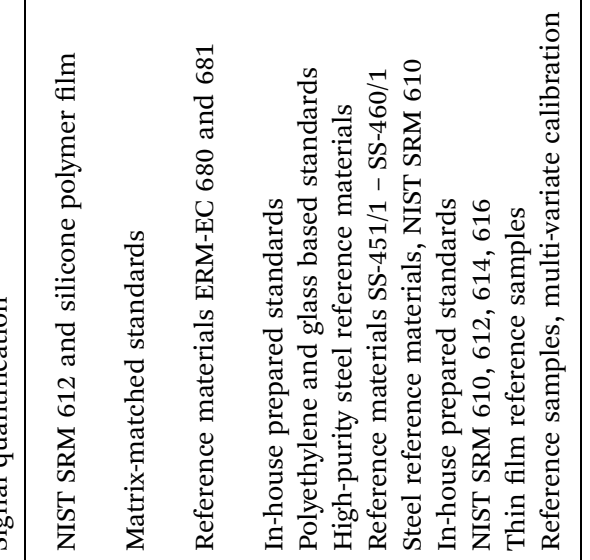

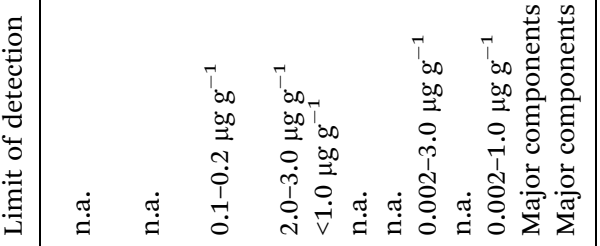

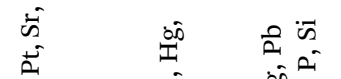

ही है वर्त

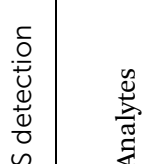

हो के के

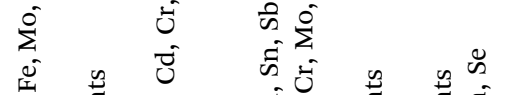

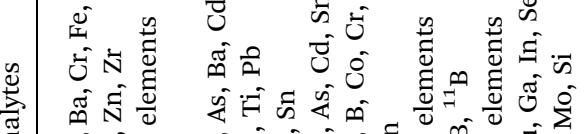

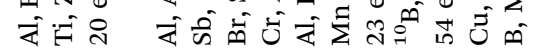

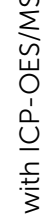

$\stackrel{\circ}{\stackrel{0}{\circ}}$

है

.$\subseteq$

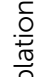

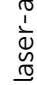

¿ั

$\frac{\tilde{a}}{\stackrel{0}{2}}$

ชิ

든

$\frac{0}{\frac{0}{0}}$

$\underset{\frac{m}{0}}{\frac{0}{\circ}}$

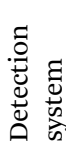

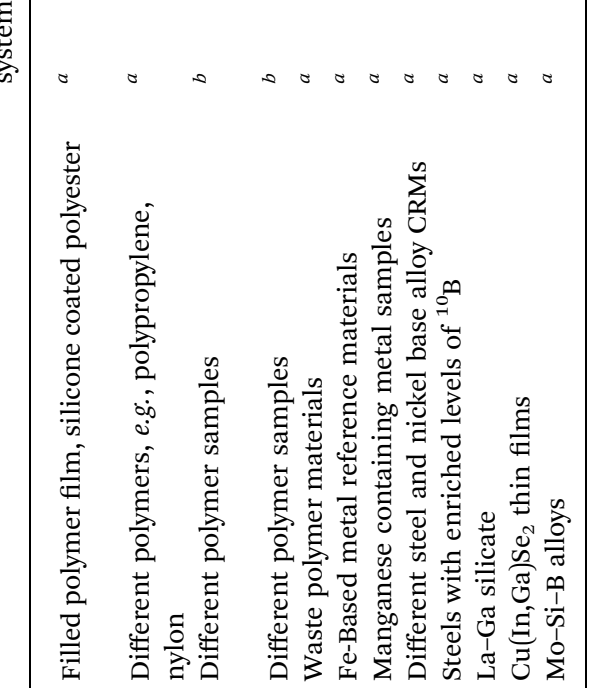

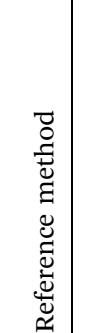

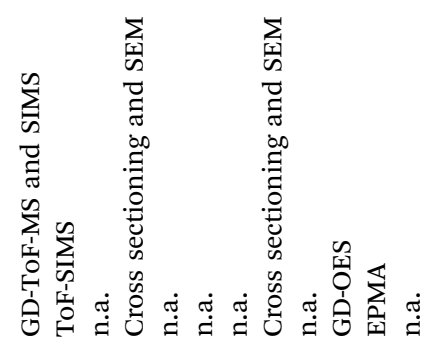

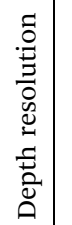

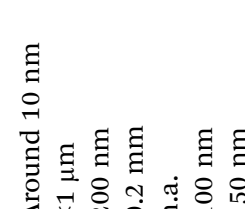

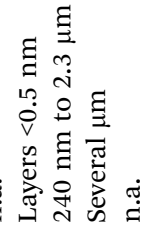

กี क

जक के

ही $\sum^{20} \bar{E}$

की

के

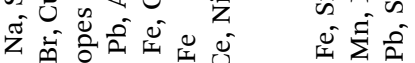

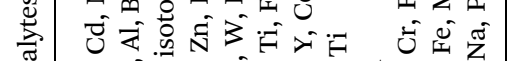

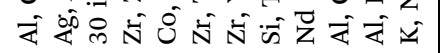

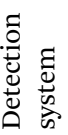

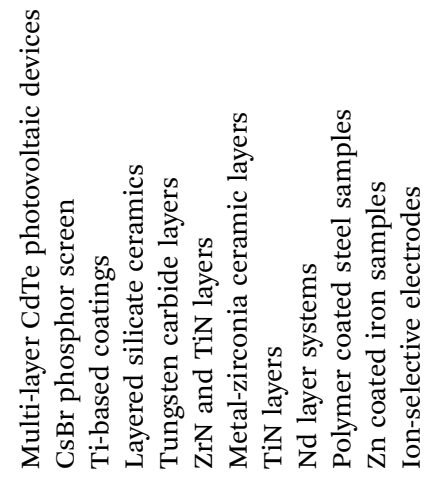

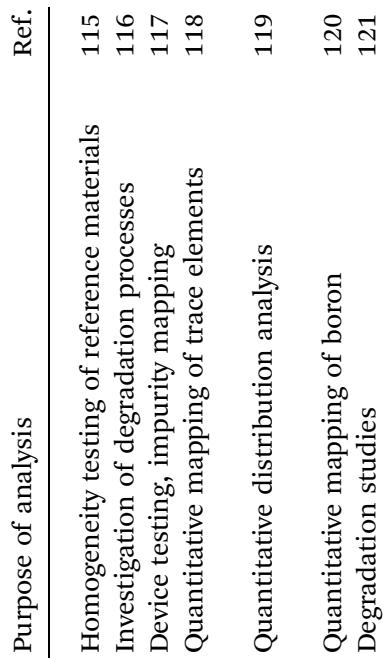

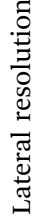

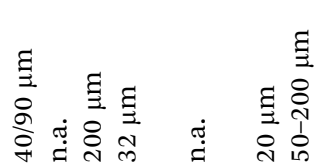

宝

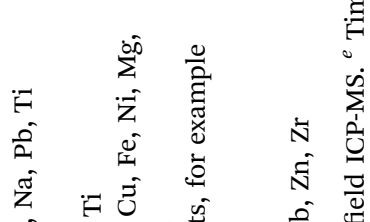

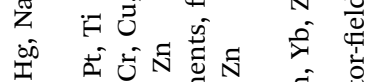

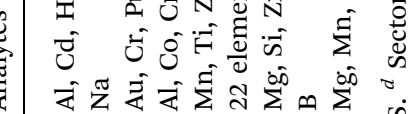

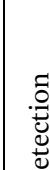

:

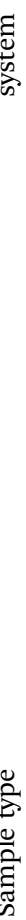

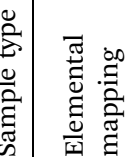

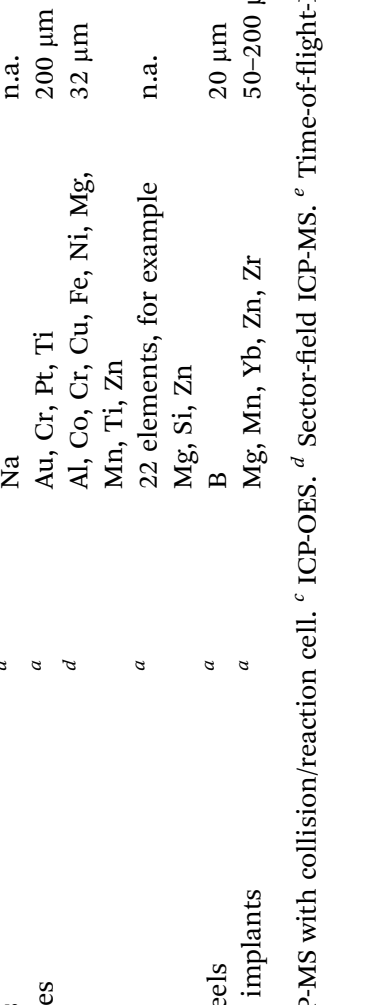

$\frac{\mathscr{U}}{3} \cdot \frac{\tilde{v}}{\mathrm{~s}}$

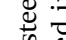

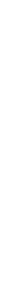

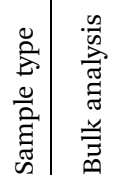

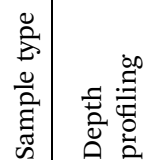

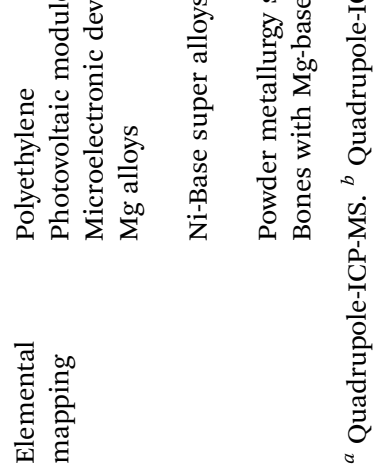


Summing up, LA-ICP-MS has promising perspectives for its use in the analysis of technological samples. Many research topics in this field or even quality control in industry could benefit from the advantages of LA-ICP-MS while keeping up the ease of use and the maintenance costs of the instrumentation compared to other analysis techniques.

\section{Conclusion \& outlook}

The use of spectrochemical techniques such as AAS, ICP-OES or ICP-MS is common in the characterization of technologically relevant materials. When compared to conventional dissolution of the solid material with subsequent analysis of the derived solution, the application of solid sampling techniques offers several advantages. The prevention of time consuming and elaborate sample pre-treatment, improvements in sensitivity of analysis and the reduction of consumed sample material are just a few examples. However, from the analytical point of view, the most important benefits are access to "insoluble samples" and the ability to perform micro-local analysis. The applicability of electro-thermal-vaporization is restricted to powdered or small compact samples. Therefore, the focus of this method is measurement of average sample concentrations. The high sensitivity and versatility which can be achieved with this technique makes it uniquely suited for the multi-element analysis of ceramics or high purity materials. Being able to analyze powders directly (without the need to compress powder into solid pellets, as required for LA analysis) is an advantage of ETV, especially in the emerging field of nano-particle analysis (i.e., for monitoring of contaminants). Laser ablation in liquid combines the strengths of solid sampling and liquid analysis. With this approach the composition of compact samples can be analyzed easily, utilizing external calibration with aqueous standards for signal calibration. Nevertheless, conventional LA with ICP-MS or ICP-OES detection is the most promising technique for the measurement of solid samples. Since sample homogeneity is an important issue for many materials, the possibility of micro-local analysis with resolutions in the low $\mu \mathrm{m}$ range with detection limits in the order of $\mu \mathrm{g} \mathrm{g}^{-1}$ is very attractive. Furthermore, LA-ICP-MS also allows gathering information about the distribution of the analytes within the sample, applications reported in the field of material characterization include elemental mapping as well as depth profile analysis. Limitations in the availability of reference materials with a multitude of certified trace elements makes quantification complicated, however, compared to well established techniques such as GD-MS or SIMS the accuracy that can be achieved without the use of matrix-matched standards is better.

Despite the benefits of the techniques reported in this work, there are some specific topics that still need further investigation in order to extend their fields of application. Since electro thermal vaporization in combination with ICP-OES or ICP-MS detection for element specific analysis is a well-established technique, no groundbreaking new developments can be expected, but there is still room for task specific improvements (e.g. new modifiers). However, the possibility to separate sample constituents in accordance to their thermal behavior offers applications which are not accessible with other solid sampling techniques. For example, tackling of non-spectral interferences (plasma-load) as well as spectral interferences arising from the sample matrix is enabled. The thermal separation of different species from the same element opens also new perspectives in the characterization of functional materials. In contrast, laser ablation offers instrumental as well as methodical developments. Ongoing improvements in laser technology combined with more sensitive detection devices will expand the capabilities of LA in the field of micro-local analysis, elemental mapping and depth profiling. High lateral resolutions in the low $\mu \mathrm{m}$ scale can be applied for example for investigations of grains structures in steels and ceramics. Analysis of thin films, coatings or multi-layer systems is another field of application. Elaborate laser systems might be able to challenge the depth resolutions typically achieved by GDMS or SIMS. With the use of improved concepts for sample preparation and advanced data treatment procedures it should be possible to overcome current limitations in signal quantification, thereby improvements in accuracy and precision of analysis could be achieved.

\section{Acknowledgements}

The research presented in this contribution was financially supported by the Austrian Science Fund (FWF): P25030-N28. M. B. wants to thank the MEIBio PhD program of the Vienna University of Technology for providing a scholarship for the period 2013-2016.

\section{References}

1 G. Friedbacher and H. Bubert, Surface and Thin Film Analysis: A Compendium of Principles, Instrumentation, and Applications, Second Edition, 2011.

2 J. S. Becker and H.-J. Dietze, Int. J. Mass Spectrom., 2003, 228, 127-150, DOI: 10.1016/S1387-3806(03)00270-7.

3 J. Pisonero, B. Fernández and D. Günther, J. Anal. At. Spectrom., 2009, 24, 1145-1160, DOI: 10.1039/b904698d.

4 B. Fernández, J. M. Costa, R. Pereiro and A. Sanz-Medel, Anal. Bioanal. Chem., 2010, 396, 15-29, DOI: 10.1007/ s00216-009-2959-6.

5 E. Vereda Alonso, A. García de Torres, M. T. Siles Cordero and J. M. Cano Pavón, Microchem. J., 2011, 97, 101-108, DOI: 10.1016/j. microc.2010.07.012.

6 M. T. Larrea, I. Gomez-pinilla and J. C. Farinas, J. Anal. At. Spectrom., 1997, 12, 1323-1332, DOI: 10.1039/A702875J.

7 M. Balcerzak, Anal. Sci., 2002, 18, 737-750, DOI: 10.2116/ analsci.18.737.

8 J. Sneddon, C. Hardaway, K. K. Bobbadi and A. K. Reddy, Appl. Spectrosc. Rev., 2006, 41, 1-14, DOI: 10.1080/ 05704920500385445.

9 Z. Wang, D. Qiu, G. Tao and P. Yang, J. Anal. At. Spectrom., 2009, 24, 1258-1261, DOI: 10.1039/b900121b.

10 Z. Wang, J. Zhang, D. Qiu, H. Zou, H. Qu, Y. Chen and P. Yang, J. Anal. At. Spectrom., 2010, 25, 1482-1484, DOI: 10.1039/b917066a. 
11 J. Hassler, P. Barth, S. Richter and R. Matschat, J. Anal. At. Spectrom., 2011, 26, 2404-2418, DOI: 10.1039/c1ja10149h.

12 D. Bauer, T. Vogt, M. Klinger, P. J. Masset and M. Otto, Anal. Chem., 2014, 86, 10380-10388, DOI: 10.1021/ac502823e.

13 M. P. Mateo, C. C. Garcia and R. Hergenröder, Anal. Chem., 2007, 79, 4908-4914, DOI: 10.1021/ac070241q.

14 B. Hattendorf, J. Pisonero, D. Günther and N. Bordel, Anal. Chem., 2012, 84, 8771-8776, DOI: 10.1021/ac302137x.

15 L. E. S. Ebdon, M. Foulkes and K. Sutton, J. Anal. At. Spectrom., 1997, 12, 213-229, DOI: 10.1039/A604914A.

16 M. C. Santos and J. A. Nóbrega, Appl. Spectrosc. Rev., 2006, 41, 427-448, DOI: 10.1080/05704920600726191.

17 M. A. Belarra, M. Resano, F. Vanhaecke and L. Moens, TrAC, Trends Anal. Chem., 2002, 21, 828-839, DOI: 10.1016/S01659936(02)01206-2.

18 M. Resano, F. Vanhaecke and M. T. C. de Loos-Vollebregt, J. Anal. At. Spectrom., 2008, 23, 1450-1475, DOI: 10.1039/ B807756H.

19 R. E. Russo, X. Mao, H. Liu, J. Gonzalez and S. S. Mao, Talanta, 2002, 57, 425-451, DOI: 10.1016/S0039-9140(02) 00053-X.

20 C. C. Garcia, H. Lindner and K. Niemax, J. Anal. At. Spectrom., 2009, 24, 14-26, DOI: 10.1039/B813124B.

21 N. S. Mokgalaka and J. L. Gardea-Torresdey, Appl. Spectrosc. Rev., 2006, 41, 131-150, DOI: 10.1080/05704920500510703.

22 D. Günther and B. Hattendorf, TrAC, Trends Anal. Chem., 2005, 24, 255-265, DOI: 10.1016/j.trac.2004.11.017.

23 Z. Wang and P. Yang, J. Anal. At. Spectrom., 2014, 29, 20912103, DOI: 10.1039/c4ja00161c.

24 M. Aramendía, M. Resano and F. Vanhaecke, Anal. Chim. Acta, 2009, 648, 23-44, DOI: 10.1016/j.aca.2009.06.027.

25 I. B. Brenner and A. T. Zander, Spectrochim. Acta, Part B, 2000, 55, 1195-1240, DOI: 10.1016/S0584-8547(00)00243-3.

26 D. Beauchemin, Anal. Chem., 2002, 74, 2873-2894, DOI: 10.1021/ac020254y.

27 J. M. Mermet, J. Anal. At. Spectrom., 2005, 20, 11-16, DOI: 10.1039/B416511J.

28 E. S. Chaves, S. Compernolle, M. Aramendia, E. Javierre, E. Tresaco, M. T. C. de Loos-Vollebregt, A. J. Curtius and F. Vanhaecke, J. Anal. At. Spectrom., 2011, 26, 1833-1840, DOI: 10.1039/C1JA10083A.

29 M. Tanner and D. Günther, Anal. Chim. Acta, 2009, 633, 1928, DOI: 10.1016/j.aca.2008.11.041.

30 D. Langer and J. A. Holcombe, Appl. Spectrosc., 1999, 53, 1244-1250.

31 J. D. Venable, D. Langer and J. A. Holcombe, Anal. Chem., 2002, 74, 3744-3753, DOI: 10.1021/ac0110303.

32 A. Hineman and C. Stephan, J. Anal. At. Spectrom., 2014, 29, 1252-1257, DOI: 10.1039/C4JA00097H.

33 F. Laborda, E. Bolea and J. Jiménez-Lamana, Anal. Chem., 2014, 86, 2270-2278, DOI: 10.1021/ac402980q.

34 F. Vanhaecke, L. Moens, R. Dams, L. Allen and S. Georgitis, Anal. Chem., 1999, 71, 3297-3303, DOI: 10.1021/ac990016b.

35 D. P. Myers, G. Li, P. P. Mahoney and G. M. Hieftje, J. Am. Soc. Mass Spectrom., 1995, 6, 411-427, DOI: 10.1016/10440305(95)00027-B.
36 D. Tanner Scott, R. Bandura Dmitry, O. Ornatsky, I. Baranov Vladimir, M. Nitz and M. A. Winnik, in Pure and Applied Chemistry, 2008, vol. 80, p. 2627.

37 O. Borovinskaya, B. Hattendorf, M. Tanner, S. Gschwind and D. Gunther, J. Anal. At. Spectrom., 2013, 28, 226-233, DOI: $10.1039 /$ C2JA30227F.

38 D. R. Bandura, V. I. Baranov, O. I. Ornatsky, A. Antonov, R. Kinach, X. Lou, S. Pavlov, S. Vorobiev, J. E. Dick and S. D. Tanner, Anal. Chem., 2009, 81, 6813-6822, DOI: 10.1021/ac901049w.

39 O. Borovinskaya, S. Gschwind, B. Hattendorf, M. Tanner and D. Günther, Anal. Chem., 2014, 86, 8142-8148, DOI: 10.1021/ac501150c.

40 P. F. Rodríguez, J. M. Marchante-Gayón and A. Sanz-Medel, Talanta, 2006, 68, 869-875, DOI: 10.1016/ j.talanta.2005.06.006.

41 M.-L. Lin and S.-J. Jiang, J. Anal. At. Spectrom., 2011, 26, 1813, DOI: 10.1039/c1ja10100e.

42 M. Resano, M. Aramendia, W. Devos and F. Vanhaecke, J. Anal. At. Spectrom., 2006, 21, 891-898, DOI: 10.1039/b602606k.

43 H. Kataoka, Y. Okamoto, T. Matsushita, S. Tsukahara, T. Fujiwara and K. Wagatsuma, J. Anal. At. Spectrom., 2008, 23, 1108-1111, DOI: 10.1039/B803995J.

44 S. Okabayashi, S. Sakata and T. Hirata, Anal. Chim. Acta, 2015, 853, 469-476, DOI: 10.1016/j.aca.2014.11.002.

45 A. Asfaw, G. Wibetoe and D. Beauchemin, J. Anal. At. Spectrom., 2012, 27, 1928, DOI: 10.1039/c2ja30193h.

46 J. Hassler, G. Záray, K. Schwetz and K. Flórián, J. Anal. At. Spectrom., 2005, 20, 954, DOI: 10.1039/b504613k.

47 K. Nakata, B. Hashimoto, H. Uchihara, Y. Okamoto, S. Ishizaka and T. Fujiwara, Talanta, 2015, 138, 279-284, DOI: 10.1016/j.talanta.2015.03.019.

48 J. C. Farinas, R. Moreno and J.-M. Mermet, J. Anal. At. Spectrom., 1994, 9, 841-849, DOI: 10.1039/JA9940900841.

49 G. Xiang, Z. Jiang, M. He and B. Hu, Spectrochim. Acta, Part B, 2005, 60, 1342-1348, DOI: 10.1016/j.sab.2005.07.005.

50 J. S. d. Gois, É. R. Pereira, B. Welz and D. L. G. Borges, Anal. Chim. Acta, 2014, 852, 82-87, DOI: 10.1016/ j.aca.2014.09.019.

51 F. Börno, S. Richter, D. Deiting, N. Jakubowski and U. Panne, J. Anal. At. Spectrom., 2015, 30, 1064-1071, DOI: 10.1039/c4ja00442f.

52 P.-C. Li and S.-J. Jiang, Anal. Bioanal. Chem., 2006, 385, 1092-1097, DOI: 10.1007/s00216-006-0547-6.

53 C.-C. Chen, S.-J. Jiang and A. C. Sahayam, Talanta, 2015, 131, 585-589, DOI: 10.1016/j.talanta.2014.08.034.

54 R. Matschat, J. Hassler, H. Traub and A. Dette, Anal. Bioanal. Chem., 2005, 383, 1060-1074, DOI: 10.1007/ s00216-005-3415-x.

55 P. Barth, J. Hassler, I. Kudrik and V. Krivan, Spectrochim. Acta, Part B, 2007, 62, 924-932, DOI: 10.1016/ j.sab.2007.03.012.

56 F. G. Antes, E. Dullius, A. B. da Costa, R. F. Molz, J. N. G. Paniz, E. M. M. Flores and V. L. Dressler, Microchem. J., 2013, 109, 117-121, DOI: 10.1016/ j.microc.2012.03.027. 
57 T. Vogt, D. Bauer, M. Neuroth and M. Otto, Fuel, 2015, 152, 96-102, DOI: 10.1016/j.fuel.2014.12.057.

58 T. Vogt, D. Bauer, D. Nennstiel and M. Otto, Anal. Chem., 2015, 87, 10414-10420, DOI: 10.1021/ acs.analchem.5b02530.

59 D. Bauer, T. Vogt, F. Lehmann and M. Otto, Chem. Ing. Tech., 2014, 86, 1806-1811, DOI: 10.1002/cite.201400020.

60 J. Hassler, R. Matschat, S. Richter, P. Barth, A. K. Detcheva and H.-J. Waarlo, J. Anal. At. Spectrom., 2016, 31, 642-657, DOI: 10.1039/c5ja00240k.

61 S. Kaczala, A. B. Costa, E. L. Posselt, J. S. Barin, E. M. M. Flores and V. L. Dressler, J. Braz. Chem. Soc., 2015, 26, 475-483, DOI: 10.5935/0103-5053.20150300.

62 Y. Okamoto, H. Komori, H. Kataoka, S. Tsukahara and T. Fujiwara, Rapid Commun. Mass Spectrom., 2010, 24, 1265-1270, DOI: 10.1002/rcm.4508.

63 G. Xiang, B. Hu, Z. Jiang and C. Gong, J. Mass Spectrom., 2006, 41, 1378-1385, DOI: 10.1002/jms.1111.

64 B. U. Peschel, W. Herdering and J. A. C. Broekaert, Spectrochim. Acta, Part B, 2007, 62, 109-115, DOI: 10.1016/ j.sab.2007.01.006.

65 G. Ertas and J. A. Holcombe, Spectrochim. Acta, Part B, 2003, 58, 1597-1612, DOI: 10.1016/S0584-8547(03)00134-4.

66 M. Aramendía, M. Resano and F. Vanhaecke, J. Anal. At. Spectrom., 2009, 24, 41-50, DOI: 10.1039/b808951e.

67 F. Vanhaecke, M. Resano and L. Moens, Anal. Bioanal. Chem., 2002, 374, 188-195, DOI: 10.1007/s00216-002-13383.

68 I. Gelaude, R. Dams, M. Resano, F. Vanhaecke and L. Moens, Anal. Chem., 2002, 74, 3833-3842, DOI: 10.1021/ ac020060i.

69 A. Detcheva, J. Hassler and R. Georgieva, Anal. Lett., 2012, 45, 603-612, DOI: 10.1080/00032719.2011.649463.

70 P. A. Mello, M. F. Pedrotti, S. M. Cruz, E. I. Muller, V. L. Dressler and E. M. M. Flores, J. Anal. At. Spectrom., 2015, 30, 2048-2055, DOI: 10.1039/c5ja00136f.

71 B. U. Peschel, F. Andrade, W. C. Wetzel, G. D. Schilling, G. M. Hieftje, J. A. C. Broekaert, R. Sperline, M. B. Denton, C. J. Barinaga and D. W. Koppenaal, Spectrochim. Acta, Part B, 2006, 61, 42-49, DOI: 10.1016/ j.sab.2005.11.007.

72 M. A. Amberger and J. A. C. Broekaert, J. Anal. At. Spectrom., 2010, 25, 1308, DOI: 10.1039/c003290e.

73 P.-K. Hsiao, S.-J. Jiang and A. C. Sahayam, J. Anal. At. Spectrom., 2011, 26, 586, DOI: 10.1039/c0ja00203h.

74 D. L. G. Borges, B. Welz and A. José Curtius, Microchim. Acta, 2007, 159, 19-26, DOI: 10.1007/s00604-006-0730-7.

75 R. E. Russo, X. Mao, J. J. Gonzalez, V. Zorba and J. Yoo, Anal. Chem., 2013, 85, 6162-6177, DOI: 10.1021/ac4005327.

76 R. E. Russo, X. Mao and S. S. Mao, Anal. Chem., 2002, 74, 70A-77A.

77 A. Limbeck, P. Galler, M. Bonta, G. Bauer, W. Nischkauer and F. Vanhaecke, Anal. Bioanal. Chem., 2015, 407, 65936617, DOI: 10.1007/s00216-015-8858-0.

78 J. Vogl, D. Liesegang, M. Ostermann, J. Diemer, M. Berglund, C. R. Quétel, P. D. P. Taylor and K. G. Heumann, Accredit. Qual. Assur., 2000, 5, 314-324.
79 R. E. Wolf, C. Thomas and A. Bohlke, Appl. Surf. Sci., 1998, 127-129, 299-303.

80 J. Marshall, J. Franks, I. Abell and C. Tye, J. Anal. At. Spectrom., 1991, 6, 145-150, DOI: 10.1039/JA9910600145.

81 M. Resano, E. García-Ruiz and F. Vanhaecke, Spectrochim. Acta, Part B, 2005, 60, 1472-1481, DOI: 10.1016/ j.sab.2005.09.006.

82 B. Izgi and M. Kayar, Talanta, 2015, 139, 117-122, DOI: 10.1016/j.talanta.2015.02.042.

83 T. Stehrer, J. Heitz, J. D. Pedarnig, N. Huber, B. Aeschlimann, D. Günther, H. Scherndl, T. Linsmeyer, H. Wolfmeir and E. Arenholz, Anal. Bioanal. Chem., 2010, 398, 415-424, DOI: 10.1007/s00216-010-3963-6.

84 D. A. Melford, Philos. Trans. R. Soc. London, Ser. A, 1980, 295, 89-103, DOI: 10.1098/rsta.1980.0078.

85 H. Yasuhara, T. Okano and Y. Matsumura, Analyst, 1992, 117, 395-399, DOI: 10.1039/AN9921700395.

86 R. Usero, A. G. Coedo, M. T. Dorado and I. Padilla, Appl. Spectrosc., 2009, 63, 859-864.

87 H. Wiltsche and D. Günther, Anal. Bioanal. Chem., 2011, 399, 2167-2174, DOI: 10.1007/s00216-010-4605-8.

88 C. Kurta, L. Dorta, F. Mittermayr, K. Prattes, B. Hattendorf, D. Günther and W. Goessler, J. Anal. At. Spectrom., 2014, 29, 185-192, DOI: 10.1039/c3ja50194a.

89 V. A. Khvostikov, V. K. Karandashev, Z. P. Burmii and O. A. Buzanov, J. Anal. Chem., 2014, 69, 495-501, DOI: 10.1134/S1061934814030083.

90 S. Lee, J. J. Gonzalez, J. H. Yoo, J. R. Chirinos, R. E. Russo and S. Jeong, Thin Solid Films, 2015, 577, 82-87, DOI: 10.1016/j.tsf.2015.01.026.

91 A. Cakara, M. Bonta, H. Riedl, P. Mayerhofer and A. Limbeck, Spectrochim. Acta, Part B, 2015, 120, 57-62, DOI: 10.1016/j.sab.2016.04.004.

92 E. V. Muravitskaya, V. A. Rosantsev, M. V. Belkov, E. A. Ershov-Pavlov and E. V. Klyachkovskaya, Spectrochim. Acta, Part B, 2009, 64, 119-125, DOI: 10.1016/ j.sab.2008.11.003.

93 G. W. Yang, Prog. Mater. Sci., 2007, 52, 648-698, DOI: 10.1016/j.pmatsci.2006.10.016.

94 Z. Yan and D. B. Chrisey, J. Photochem. Photobiol., C, 2012, 13, 204-223, DOI: 10.1016/j.jphotochemrev.2012.04.004.

95 R. Castell, E. D. Greaves, L. Abdala and H. Barros, Appl. Phys. B: Lasers Opt., 2012, 109, 47-53, DOI: 10.1007/ s00340-012-5175-6.

96 D. N. Douglas, J. L. Crisp, H. J. Reid and B. L. Sharp, J. Anal. At. Spectrom., 2011, 26, 1294-1301, DOI: 10.1039/ C0JA00144A.

97 S. Okabayashi, T. D. Yokoyama, Y. Kon, S. Yamamoto, T. Yokoyama and T. Hirata, J. Anal. At. Spectrom., 2011, 26, 1393-1400, DOI: 10.1039/C0JA00200C.

98 R. Machida, T. Nakazawa, Y. Sakuraba, M. Fujiwara and N. Furuta, J. Anal. At. Spectrom., 2015, 30, 2412-2419, DOI: 10.1039/C5JA00349K.

99 R. Escobar Galindo, R. Gago, J. Albella and A. Lousa, $\operatorname{Tr} A C$, Trends Anal. Chem., 2009, 28, 494-505.

100 D. Günther, R. Frischknecht, C. A. Heinrich and H. J. Kahlert, J. Anal. At. Spectrom., 1997, 12, 939-944. 
101 A. Gutiérrez-González, C. González-Gago, J. Pisonero, N. Tibbetts, A. Menéndez, M. Vélez and N. Bordel, J. Anal. At. Spectrom., 2015, 30, 191-197, DOI: 10.1039/c4ja00196f.

102 L. I. L. Balcaen, J. Lenaerts, L. Moens and F. Vanhaecke, J. Anal. At. Spectrom., 2005, 20, 417-423, DOI: 10.1039/ b412287a.

103 D. Bleiner, A. Plotnikov, C. Vogt, K. Wetzig and D. Günther, Fresenius. J. Anal. Chem., 2000, 368, 221-226, DOI: 10.1007/ s002160000417.

104 A. Hrdlička, V. Otruba, K. Novotný, D. Günther and V. Kanický, Spectrochim. Acta, Part B, 2005, 60, 307-318, DOI: 10.1016/j.sab.2005.02.001.

105 J. S. Lee and H. B. Lim, Appl. Surf. Sci., 2015, 327, 483-489, DOI: 10.1016/j.apsusc.2014.11.123.

106 V. Kanicky, V. Otruba and J. M. Mermet, Spectrochim. Acta, Part B, 2000, 55, 575-586, DOI: 10.1016/S0584-8547(00) 00167-1.

107 V. Kanicky, I. Novotny, J. Musil and J.-M. Mermet, Appl. Spectrosc., 1997, 51, 1042-1046.

108 V. Kanicky, J. Musil and J.-M. Mermet, Appl. Spectrosc., 1997, 51, 1037-1041.

109 L. Zaoralkova, A. Hrdlicka, V. Otruba, P. Sulovsky, N. Gilon, D. Günther and V. Kanicky, Chem. Pap., 2011, 65, 769-781, DOI: 10.2478/s11696-011-0085-3.

110 A. Michalska, M. Wojciechowski, E. Bulska and K. Maksymiuk, Talanta, 2010, 82, 151-157, DOI: 10.1016/ j.talanta.2010.04.012.

111 G. M. Rupp, A. Limbeck, M. Kubicek, A. Penn, M. StogerPollach, G. Friedbacher and J. Fleig, J. Mater. Chem. A, 2014, 2, 7099-7108, DOI: 10.1039/c3ta15327d.
112 G. M. Rupp, H. Téllez, J. Druce, A. Limbeck, T. Ishihara, J. Kilner and J. Fleig, J. Mater. Chem. A, 2015, 3, 2275922769, DOI: 10.1039/c5ta05279c.

113 H. A. O. Wang, D. Grolimund, C. Giesen, C. N. Borca, J. R. H. Shaw-Stewart, B. Bodenmiller and D. Günther, Anal. Chem., 2013, 85, 10107-10116, DOI: 10.1021/ ac400996x.

114 S. J. M. Van Malderen, J. T. van Elteren and F. Vanhaecke, Anal. Chem., 2015, 87, 6125-6132, DOI: 10.1021/ acs.analchem.5b00700.

115 A. M. Dobney, A. J. G. Mank, K. H. Grobecker, P. Conneely and C. G. De Koster, Anal. Chim. Acta, 2000, 423, 9-19, DOI: 10.1016/S0003-2670(00)01047-3.

116 K. M. Stika, C. S. Westphal, J. Kapur, R. G. Raty, J. Li, J. G. Kopchick, W. J. Gambogi, B. Hamzavytehrany, A. Z. Bradley, J. R. Marsh and B. W. Foltz, 2014 IEEE 40th Photovoltaic Specialist Conference, PVSC, 2014.

117 M. V. Zoriy, D. Mayer and J. S. Becker, J. Am. Soc. Mass Spectrom., 2009, 20, 883-890, DOI: 10.1016/ j.jasms.2009.01.004.

118 C. Latkoczy, Y. Müller, P. Schmutz and D. Günther, Appl. Surf. Sci., 2005, 252, 127-132, DOI: 10.1016/ j.apsusc.2005.02.040.

119 J. Hu, M. Han, L. Yuan and H. Wang, ISIJ Int., 2012, 52, 2055-2058, DOI: 10.2355/isijinternational.52.2055.

120 C. Gierl-Mayer, V. Bauer, A. Limbeck and H. Danninger, Powder Metall. Prog., 2014, 14, 129-136.

121 J. Draxler, A. Zitek, M. Meischel, S. E. Stranzl-Tschegg, B. Mingler, E. Martinelli, A. M. Weinberg and T. Prohaska, J. Anal. At. Spectrom., 2015, 30, 2459-2468, DOI: $10.1039 /$ C5JA00354G. 